Universidade de Pernambuco

Campus Caruaru

Bacharelado em Sistemas de Informação

\title{
Modelos de Aprendizado de Máquina para Detecção de Atividades Humanas em Dados de Sensores Multimodais
}

Trabalho de Graduação

Sistemas de Informação

\author{
Silvano Ramos de Assis Neto \\ Orientadora: Prof. Dra. Patricia Takako Endo \\ Coorientador: Guto Leoni Santos
}

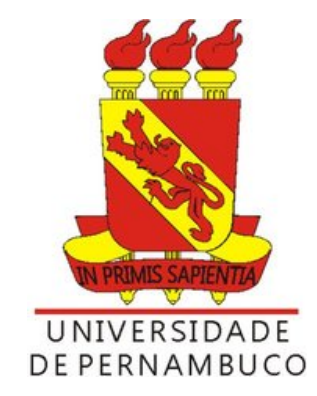




\title{
Universidade de Pernambuco
}

\author{
Silvano Ramos de Assis Neto
}

\section{Modelos de Aprendizado de Máquina para Detecção de Atividades Humanas em Dados de Sensores Multimodais}

\begin{abstract}
Monografia apresentada junto ao Curso de Sistemas de Informação da Universidade de Pernambuco, campus Caruaru, como requisição parcial à obtenção do título de Bacharel.
\end{abstract}

Orientador: Patricia Takako Endo

Coorientador: Guto Leoni Santos

\author{
Universidade de Pernambuco - UPE \\ Campus Caruaru \\ Graduação
}

Caruaru

2019 
Dados internacionais de Catalogação-na-Publicação (CIP)

Universidade de Pernambuco - Campus Caruaru

Unidade de Ensino Gov. Miguel Arraes de Alencar

N469m Neto Assis, Silvano Ramos

Modelos de aprendizados de máquina para detecção de em atividades humanas de dados de sensores multimodais / Silvano Ramos Neto Assis, Caruaru, 2019.

$47 \mathrm{f}$ :

Orientadora: Profa. Dra. Patrícia Takako Endo.

Graduação (Bacharelado em Sistemas de Informação) - Universidade de Pernambuco, Caruaru, 2018.

1 INTELIGÊNCIA ARTIFICIAL 2 SENSORES MULTIMODAIS 3 ATIVIDADES HUMANAS 4 INOVAÇÃO. Endo, Patrícia Takako (orient.) II Título

CDD $23^{\text {th }}$ ed. -006.3

Elane Cristina de Oliveira - CRB4/1875 
Monografia de Graduaçáo apresentada por Silvano Ramos de Assis Neto do Curso de Gradueçato em Sistemas de Informaçāo da Universidade de Pernambuco, campus Caruaru, sob o titulo "Modelos de Aprendizado de Máquina para Detecçăo de Atividndes Humanas em Dados de Sensores Multimodals", orientada pela Profa. Dra. Patricla Takako Endo, coorientada pelo Me. Guto Leoni Santos e aprovada pela Banca Examinadora formada por:

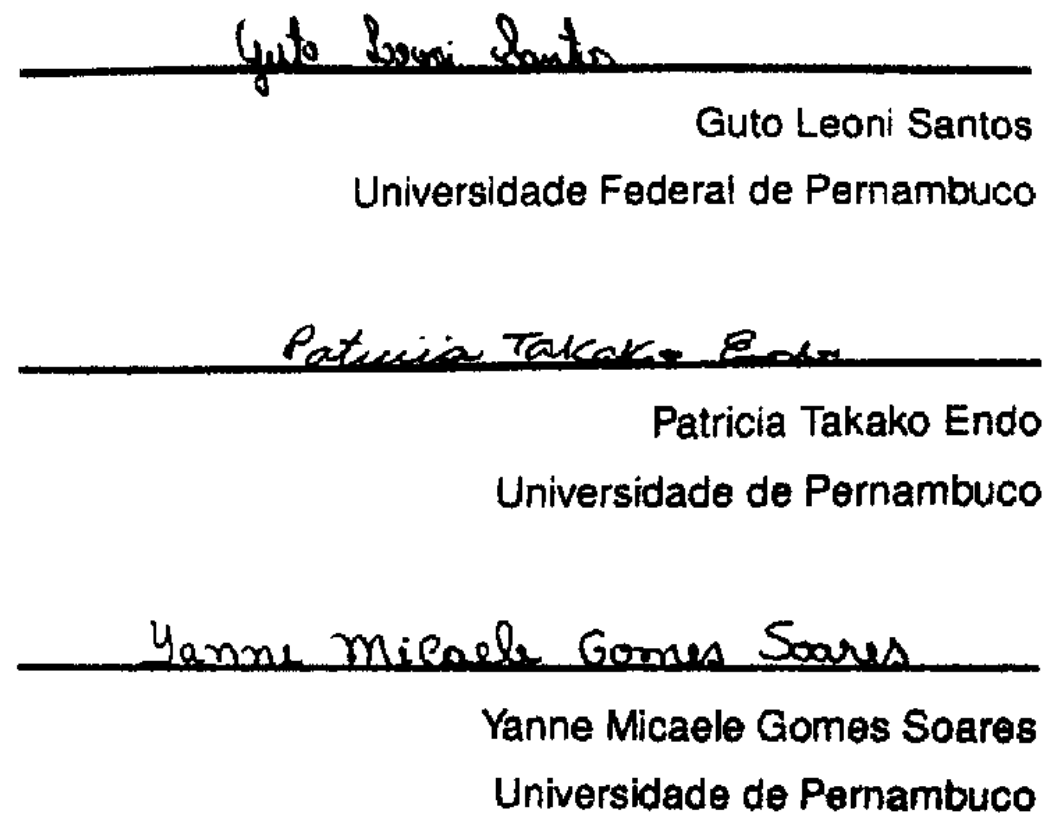

Visto e permitida a impressāo.

Caruaru, 02 de julho de 2019.

Prof*. Dra. Mirele Moutinho Lima

Coordenadora do Curso de Bacharelado em Sistemas de Informação da Universidade de Pernambuco - Campus Caruaru. 
Este trabalho é dedicado a fé e amor próprios. 


\section{Agradecimentos}

Primeiramente agradeço aos meus pais, que concederam de sua energia e suor para que me houvesse a possibilidade de cursar numa universidade. Considerome um dos poucos privilegiados com a oportunidade, num país marcado pela desigualdade social e pelo desinteresse em educação do atual governo deste país.

Agradeço a Dayvid Clementino, João Alexandre, Lucas Feijó, Matheus Ferreira e Wedson Soares pela colaboração e parceria durante os anos letivos, parceria esta que vem se estendendo fora da academia e que espero manter por muitos anos. Obrigado por me ajudarem a lutar contra minhas neuras e me motivar a fazer mais do que eu poderia imaginar que conseguiria.

Devo também o agradecimento a todos os professores aos quais pude desfrutar da experiência e vivenciar construção de conhecimento, em especial Dra. Mirele Moutinho, Dra. Patricia Endo, Dr. Jorge Fonsêca, Dr. Wylliams Barbosa, Dr. Thiago Farias e o Mestre Alex Borges. Além deles, agradeço também ao meu co-orientador Mestre Guto Leoni, pelos direcionamentos técnicos e palavras de amizade que culminaram no esforços dedicados a este trabalho. 
"Concentre todos seus pensamentos na tarefa que está realizando. Os raios de sol não queimam até que sejam colocados em foco." Alexander Graham Bell 


\section{Resumo}

O reconhecimento de atividades humanas (RAH) atraiu a atenção de pesquisadores em diversas áreas. No âmbito da saúde ele pode contribuir para prevenção de quedas, que são uma das principais causas de lesões fatais não intencionais em todo o mundo. Atrelado a isto, há o crescente interesse no estudo e desenvolvimento de modelos de aprendizado voltados para RAH, capazes de extrair informação a partir de dados de múltiplas modalidades. Diante do escopo limitado dos trabalhos de pesquisa observados na literatura, este trabalho desenvolveu, avaliou e comparou 4 modelos classificadores (Bi-LSTM, Bi-GRU, Random Forest com OvR e Gradient Boost Machine com OvR), capazes de classificar a 12 tipos de atividades humanas a partir de dados multimodais. Os dados foram providos por sensores de múltiplas modalidades, sejam vestíveis (acelerômetros, giroscópios, etc.) ou de ambiente (sensores infravermelho e câmeras), através do experimento com 9 pessoas. Após coletados, os dados foram disponibilizados pela Universidad Panamericana para a competição Challenge $U P$, voltada a RAH e detecção de quedas. Modelos neurais alcançaram até $94 \%$ de acurácia e 70,6\% de F1-score pelo Bi-LSTM, enquanto modelos baseados em algoritmos tradicionais de aprendizado alcançaram acurácia de até 99,4\% e F1-score de até 95,9\% pelo Gradient Boost Machine com OvR.

Palavras-chaves: Reconhecimento de Atividades Humanas, Sensores Multimodais, Aprendizagem de Máquina 


\section{Abstract}

The human activity recognition (HAR) attracted the attention of researchers in several areas. In the field of health it can contribute to the prevention of falls, which are a major cause of unintentional fatal injuries worldwide. Coupled with this, there is a growing interest in the study and development of HAR learning models capable of extracting information from multiple modal data. Considering the limited scope of the research works observed in the literature, this work developed, evaluated, and compared 4 classifier models (Bi-LSTM, Bi-GRU, Random Forest with OvR and Gradient Boost Machine with OvR) capable of attending to 12 types of human activities from multimodal data. The data were provided by sensors of multiple modalities, either wearable (accelerometers, gyroscopes, etc.) or environment (infrared sensors and cameras), through the experiment with 9 people. After being collected, the data were made available by Universidad Panamericana for the Challenge UP competition, focused on RAH and detection of falls. Neural models achieved up to $94 \%$ accuracy and $70.6 \% \mathrm{~F} 1$-score by Bi-LSTM, while models based on traditional learning algorithms achieved accuracy of up to $99.4 \%$ and F1-score of up to $95.9 \%$ by Gradient Boost Machine with OvR.

Key-words: Human Activity Recognition, Multimodal Sensors, Machine Learning 


\section{Lista de ilustrações}

Figura 1 - Exemplo de estrutura de uma rede neural artificial . . . . . . . 18

Figura 2 - Exemplo de estrutura de uma rede neural recorrente . . . . . . . . 18

Figura 3 - Estrutura das células das unidades LSTM e GRU - Fonte: (Michael Nguyen, 2018) . . . . . . . . . . . . . . . . . . . . 20

Figura 4 - Computação de uma RNN bidirecional - Fonte: (GOODFELLOW et al., 2016) . . . . . . . . . . . . . . . . . . . . 22

Figura 5 - Exemplo de estrutura de uma árvore de decisão . . . . . . . . . 22

Figura 6 - Exemplo de conjunto de árvores de decisão de uma Random Forest 23

Figura 7 - Distribuição dos sensores usados para coletar os dados: (a) Sensores vestíveis e headset EEG localizados no corpo humano, e (b) Layout dos sensores infravermelho e câmeras - Fonte: (CHALLENGE..., 2019). . . . . . . . . . . . . . . . . . . . 27

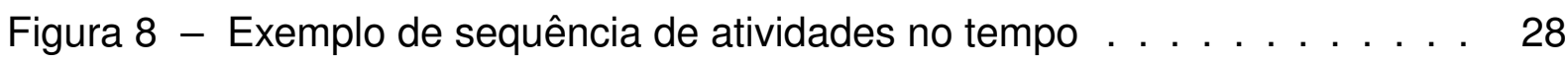

Figura 9 - Modelo LSTM Bidirecional . . . . . . . . . . . . . . . . . . 30

Figura 10 - Acurácia do modelo LSTM Bidirecional através das épocas . . . . 30

Figura 11 - Modelo GRU Bidirecional . . . . . . . . . . . . . . . . . 31

Figura 12 - Acurácia do modelo GRU Bidirecional através das épocas . . . . . . 31

Figura 13 - Modelo Random Forest com estratégia OvR . . . . . . . . . . . . 32

Figura 14 - Modelo Gradient Boost Machine com estratégia OvR . . . . . . . . . 33

Figura 15 - Matriz de confusão - LSTM Bidirecional . . . . . . . . . . . . 37

Figura 16 - Matriz de confusão - GRU Bidirecional . . . . . . . . . . . . . 37

Figura 17 - Matriz de confusão - Random Forest com OvR . . . . . . . . . . 38

Figura 18 - Matriz de confusão - Gradient Boost Machine com OvR . . . . . . 38 


\section{Lista de tabelas}

Tabela 1 - Atividades e suas respectivas descrições . . . . . . . . . . . . 27

Tabela 2 - Canais selecionados para o treinamento dos modelos neurais . . . 29

Tabela 3 - Canais selecionados para o treinamento dos modelos baseados em árvore de decisão . . . . . . . . . . . . . . . . . . . . . 29

Tabela 4 - Taxa de precisão dos modelos por atividade . . . . . . . . . . 39

Tabela 5 - Taxa de sensibilidade dos modelos por atividade . . . . . . . . . 40

Tabela 6 - Resultados do Teste Wilcoxon para o modelo Random Forest com

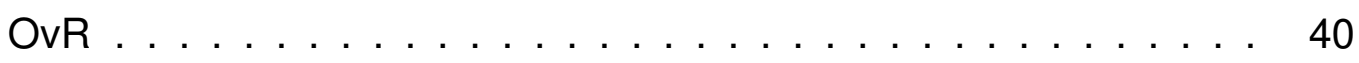

Tabela 7 - Resultados do Teste Wilcoxon para o modelo Gradient Boost Machine com OvR . . . . . . . . . . . . . . . . . . . . 40

Tabela 8 - Taxa de especificidade dos modelos por atividade . . . . . . . . 41

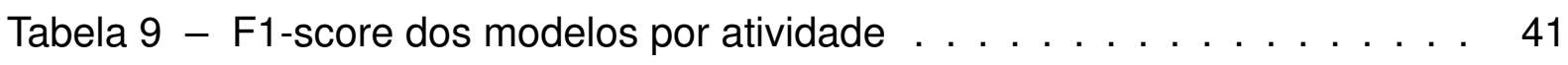

Tabela 10 - Taxas gerais das métricas por modelo . . . . . . . . . . . 41 


\section{Lista de abreviaturas e siglas}

$\begin{array}{ll}\text { Bi-GRU } & \text { Bidirectional Gated Recurrent Unit } \\ \text { Bi-LSTM } & \text { Bidirectional Long Short-Term Memory } \\ \text { CNN } & \text { Convolutional Neural Network } \\ \text { FN } & \text { Falsos Negativos } \\ \text { FP } & \text { Falsos Positivos } \\ \text { GBM } & \text { Gradient Boost Machine } \\ \text { GRU } & \text { Gated Recurrent Unit } \\ \text { IOT } & \text { Internet of Things } \\ \text { LSTM } & \text { Long Short-Term Memory } \\ \text { OMS } & \text { Organização Mundial da Saúde } \\ \text { OvR } & \text { One-versus-the-Rest } \\ \text { RAH } & \text { Reconhecimento de Atividade Humana } \\ \text { RF } & \text { Random Forest } \\ \text { RNA } & \text { Rede Neural Artificial } \\ \text { RNN } & \text { Recurrent Neural Network } \\ \text { VN } & \text { Verdadeiros Negativos } \\ \text { VP } & \text { Verdadeiros Positivos } \\ & \end{array}$




\section{Sumário}

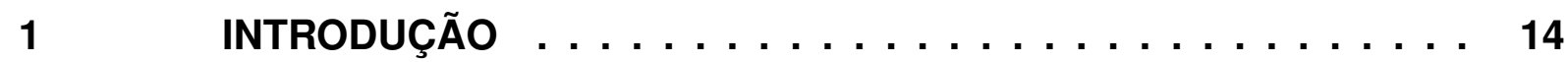

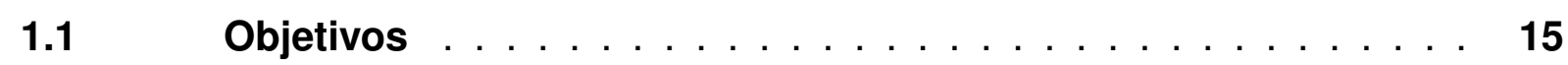

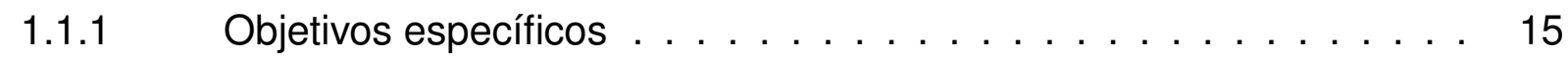

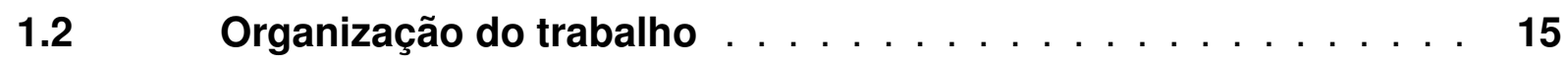

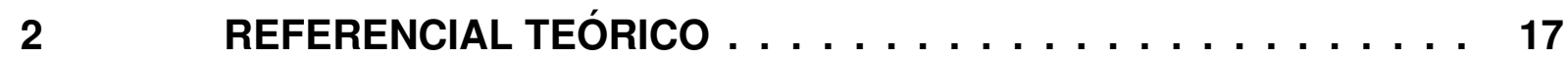

$2.1 \quad$ Técnicas para detecção de atividades humanas . . . . . . . . 17

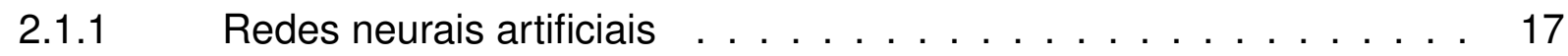

2.1.1.1 Redes Neurais Recorrentes . . . . . . . . . . . . . . . . . 18

2.1.1.2 Long Short-Term Memory (LSTM) \& Gated Recurrent Unit (GRU) . . . . 19

2.1.1.3 Bidirecionamento de redes recorrentes . . . . . . . . . . . . . 21

$2.1 .2 \quad$ Árvores de decisão . . . . . . . . . . . . . . . . . . . . 21

2.1.2.1 Random Forest . . . . . . . . . . . . . . . . 21

2.1.2.2 Gradient Boost Machine . . . . . . . . . . . . . . . . . . 22

2.1.2.3 Estratégia One-vs-the-rest $(\mathrm{OvR}) \ldots \ldots \ldots \ldots \ldots \ldots$

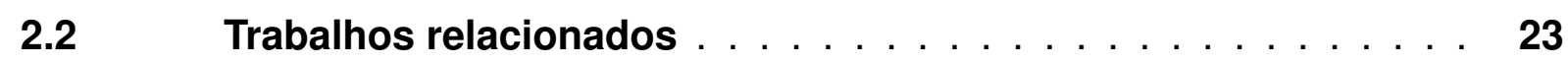

3 MODELOS PARA DETECÇÃo DE ATIVIDADES HUMANAS . . . 26

$3.1 \quad$ Cenários e data set $\ldots \ldots \ldots \ldots \ldots \ldots$

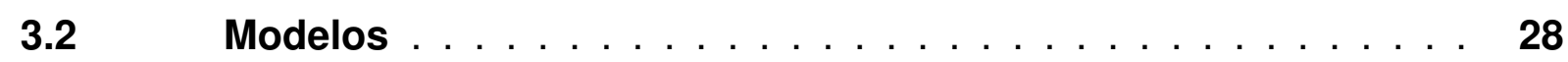

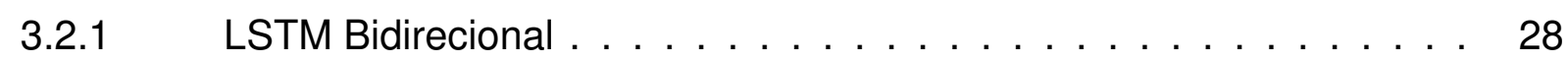

$3.2 .2 \quad$ GRU Bidirecional $\ldots \ldots \ldots \ldots$

3.2.3 Random Forest com OvR . . . . . . . . . . . . . . 31

3.2.4 Gradient Boost Machine com OvR . . . . . . . . . . . . . 32

3.2.5 Implementação dos modelos . . . . . . . . . . . . 32

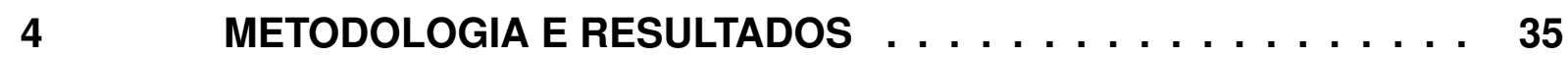

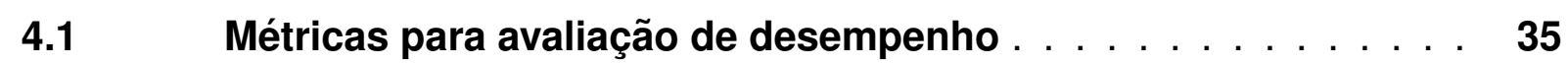

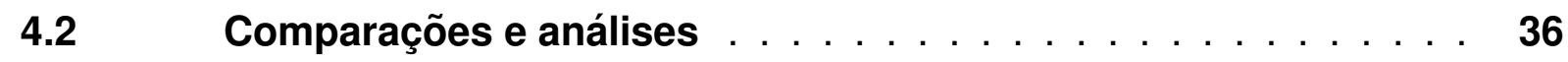

$4.2 .1 \quad$ Matriz de confusão $\ldots \ldots \ldots \ldots \ldots \ldots$

4.2 .2 Desempenho $\ldots \ldots \ldots \ldots \ldots \ldots$

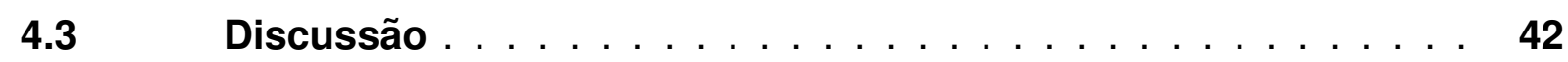

5 CONCLUSÃO E TRABALHOS FUTUROS $\ldots \ldots \ldots \ldots$

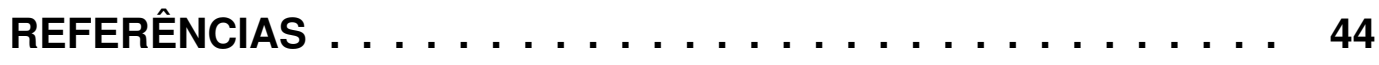


ANEXO A - UP-FALL DETECTION DATASET: DISPOSITIVOS E CANAIS $\ldots \ldots \ldots \ldots \ldots \ldots \ldots \ldots$ 


\section{Introdução}

Segundo a Organização Mundial da Saúde (OMS), cerca de 646 mil pessoas morrem por quedas anualmente, tornando-se a segunda maior causa de morte não intencional, perdendo apenas para acidentes de trânsito. Foi também constatado que pessoas acima dos 65 anos têm alto risco de morte ou de sofrer lesões graves, enquanto que crianças tornam-se um grupo de risco para lesões e morbidade devido ao estágio de desenvolvimento e associação (WORLD..., 2018). Além disso, o medo de cair pode ocasionar prejuízos indiretos a saúde, como redução ou privação de atividade física e problemas psicológicos (SCHEFFER et al., 2008).

A OMS indica também que políticas voltadas para a diminuição das taxas de injúria por queda ou lesão vem tomando ações de prevenção como: intervenções clínicas, rastreio do ambiente, avaliação de risco, orientação comportamental, fortalecimento muscular e reconversão do equilíbrio, dispositivos de apoio e programas de educação (ORGANIZATION et al., 2007). Pesquisas sugerem que sistemas para detecção de queda podem reduzir o medo de cair, impedir lesões físicas e amenizar tais consequências devido à detecção e intervenção mais rápidas (BROWNSELL; HAWLEY, 2004). Estas soluções podem se caracterizar como sistemas não vestíveis (sensíveis ao contexto) ou vestíveis (IGUAL et al., 2013).

O baixo custo dos sensores e sua integração tanto em dispositivos móveis quanto em ambientes físicos, permitiu com que pesquisadores pudessem utilizar de combinações entre dispositivos vestíveis e fontes fixas de dados de localização, no intuito de fornecer diferentes perspectivas de um dado evento ou atividade humana (NGIAM et al., 2011). Adquirir conhecimento a partir de dados multimodais não está isenta de desafios, não apenas pelo volume, variedade e velocidade característicos desses dados, mas também pela especificidade da atividade e pela eficácia da técnica utilizada para o seu reconhecimento (ROUAST et al., 2019; BALTRUŠAITIS et al., 2019; NWEKE et al., 2019; CHEN et al., 2017).

Há um foco crescente no estudo do reconhecimento de atividades humanas (RAH) baseado em abordagens de deep learning para extração de features usando dados multimodais (KANJO et al., 2019; MIOTTO et al., 2017). Uma vez que dados de modalidade única são inerentemente limitados para estudos de RAH no mundo real (CHEN et al., 2017), foram verificados também trabalhos na literatura que propuseram algoritmos de deep learning para RAH em diferentes áreas e em dados de múltiplas modalidades, como (LI et al., 2017) com Convolutional Neural Networks (CNNs), ou (ORDÓÑEZ; ROGGEN, 2016) que mescla CNN e Long Short-Term Memory (LSTM). 
Dados os cenários levantados e as limitações de escopo dos trabalhos observados na literatura, este trabalho tem a seguinte pergunta de pesquisa: "Qual ou quais modelos de aprendizagem de máquina conseguem detectar com alto desempenho atividades físicas cotidianas de através de dados multimodais?". Trabalhos como de (SUN et al., 2019) e (CHUNG et al., 2019) apontaram para o levantamento da hipótese que este trabalho de conclusão de curso lida, que é a de que deep learning possui potencial para apresentar bom desempenho na classificação de atividades a partir de dados multimodais. Entretanto, o escopo é aberto para avaliar também outros tipos de algoritmos de aprendizado de máquina, para fins de comparação.

\subsection{Objetivos}

Este trabalho tem como objetivo propor e analisar modelos de aprendizado de máquina, capazes de detectar atividades (caminhar, sentar, cair, etc.) através de dados originalmente fornecidos por sensores de diferentes modalidades (acelerômetros, giroscópios, sensores de luminosidade, etc).

\subsubsection{Objetivos específicos}

- Analisar os dados que serão utilizados como base nesse trabalho e identificar características relevantes, também chamadas de features;

- Desenvolver modelos de aprendizagem de máquina;

- Avaliar os modelos e analisar os resultados;

- Recomendar melhorias e aplicações futuras;

\subsection{Organização do trabalho}

Este trabalho de conclusão de curso está organizado em 5 partes. A seguir uma breve descrição deste e dos próximos 4 capítulos:

- Capítulo 1: o primeiro capítulo apresenta o problema em que a detecção de atividades por aprendizagem de máquina pode dar suporte, define o propósito da pesquisa e especifica quais atividades foram exercidas para alcance do objetivo geral;

- Capítulo 2: apresenta as técnicas utilizadas para construção dos modelos de aprendizado de máquina e alguns trabalhos de pesquisa relacionados à detecção de atividades humanas; 
- Capítulo 3: apresenta os classificadores desenvolvidos e os cenários em que atuaram, além de apontar as ferramentas e o ambiente utilizados para implementar e avaliar os modelos de aprendizagem;

- Capítulo 4: detalha informações sobre a base de dados utilizada para treinamento e teste dos modelos, define as métricas de avaliação, apresenta os comparativos entre os modelos e conclui com a discussão dos resultados;

- Capítulo 5: faz uma revisão geral dos insights adquiridos através deste trabalho, e mostra os próximos passos para o desenvolvimento de trabalhos futuros. 


\section{Referencial Teórico}

Esse capítulo apresenta os conceitos que fundamentam os algoritmos que integram os modelos desenvolvidos para classificação das atividades. A Seção 2.1 trata de redes neurais artificiais e suas variantes (Subseção 2.1.1), e árvores de decisão e suas variantes (Subseção 2.1.2). Na Seção 2.2 são detalhados alguns trabalhos relacionados, direcionados para detecção de atividades humanas utilizando aprendizagem de máquina.

\subsection{Técnicas para detecção de atividades humanas}

\subsubsection{Redes neurais artificiais}

Redes neurais artificiais (RNAs) são algoritmos que compõem o campo conhecido como Deep Learning, um subcampo da aprendizagem de máquina. Diferente de algoritmos de outros segmentos do aprendizado de máquina, RNAs são capazes de a partir de dados brutos extrair padrões que permitem a construção de conceitos complexos (ex.: uma cadeira numa imagem) a partir de conceitos mais simples (ex.: os bits de um pixel da cadeira na imagem) (GOODFELLOW et al., 2016).

Uma RNA se divide em 3 partes básicas, como pode-se observar no exemplo de topologia da Figura 1:

- Entrada: camada na qual os dados são introduzidos, de acordo com a dimensão pré-estabelecida pelo modelo;

- Camadas escondidas: camadas em que as features são extraídas e armazenadas;

- Saída: última camada do modelo, onde os nós calculam o valor real para cada umas das classes do modelo.

A ideia de profundidade destes algoritmos se dá pelo uso de várias camadas sucessivas e interconectadas de representações, compostas por unidades que guardam informações ou características importantes dos dados no modelo, uma alusão aos neurônios do cérebro animal (CHOLLET, 2017). Os valores que representam estas informações numa unidade neural são chamados de pesos.

Existem diversas arquiteturas que podem ser implementadas, apropriadas de acordo com o tipo do dado, como estes dados estão estruturados e qual tarefa o 
modelo precisa desempenhar. Dentre elas, Redes Neurais Recorrentes (RNN), que será descrita a seguir.

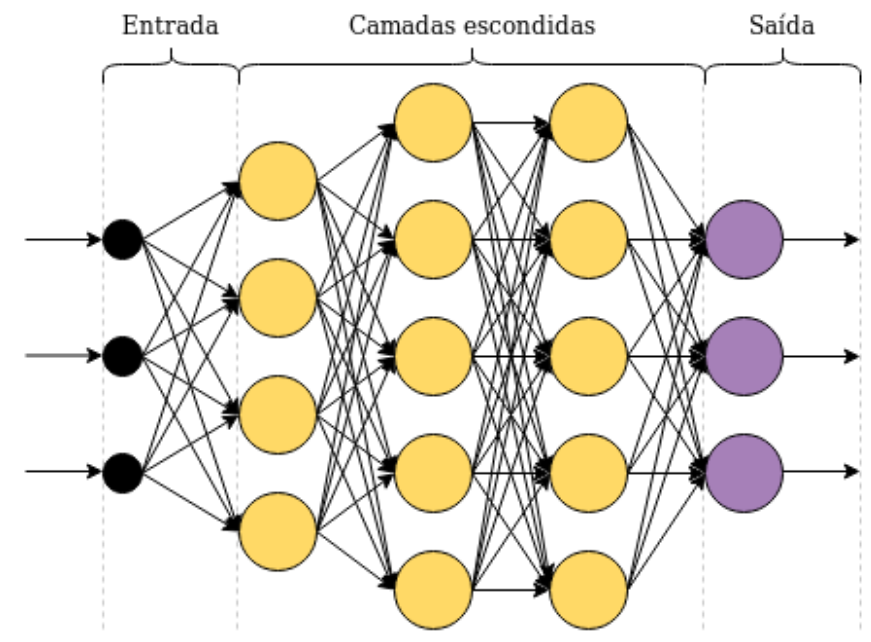

Figura 1 - Exemplo de estrutura de uma rede neural artificial

\subsubsection{Redes Neurais Recorrentes}

No caso específico das RNNs, as features entre as camadas é calculada a partir de dados sequenciais ou séries temporais. Estes dados são iterados, e para cada iteração um estado é mantido contendo informação do que foi visto na iteração anterior (CHOLLET, 2017). Em sua variante mais básica, a cada série processada o estado logo é resetado. Além disso, sua arquitetura possui um loop interno, como pode ser visto na Figura 2.

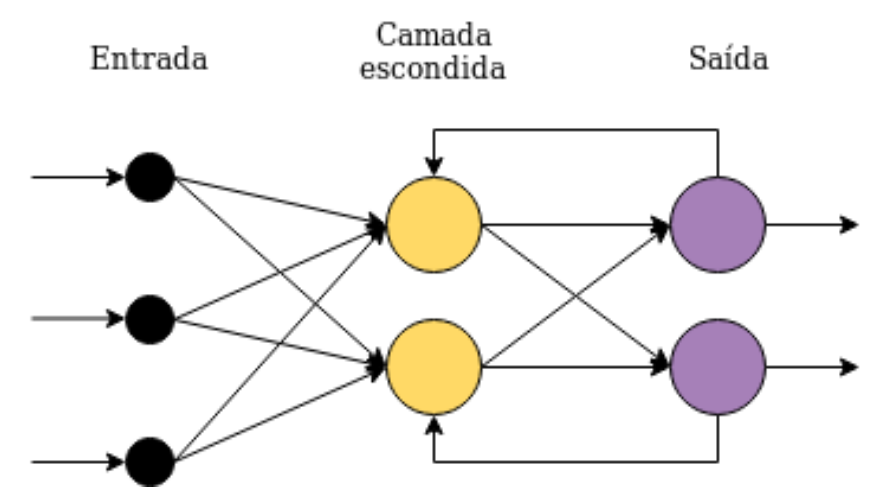

Figura 2 - Exemplo de estrutura de uma rede neural recorrente

Segundo (GOODFELLOW et al., 2016), um dos problemas apresentados por modelos neurais é a dificuldade de melhorar o desempenho de redes com muitas camadas. Em modelos tradicionais de RNN, uma mesma operação é realizada diversas vezes para cada passo de tempo. Quando o valor bruto repassado de uma unidade a outra da rede for muito pequeno, ele tenderá a desaparecer da rede, causando a perda 
de informação (vanishing). Entretanto, existem variantes da RNN tradicional que ataca esse problema. A seguir, são mostrados dois exemplos destes algoritmos.

\subsubsection{Long Short-Term Memory (LSTM) \& Gated Recurrent Unit (GRU)}

A LSTM é uma RNN capaz de salvar informação através de muitos passos de tempo, a fim de prevenir que esta não se perca pela interferência de sinais oriundos de outras unidades da rede (CHOLLET, 2017). Uma célula LSTM pode se resumir em quatro partes:

1. Forget gate: decide o quanto da informação é relevante manter, esta provida pela entrada atual $x_{t}$ e pelo estado oculto da célula anterior $h_{t-1}$. Esta informação é passada para a função (gate) sigmóide $\sigma_{g}$, denominada de função de ativação recorrente;

$$
f_{t}=\sigma_{g}\left(W_{f} x_{t}+U_{f} h_{t-1}\right)
$$

onde $W$ e $U$ são as matrizes de pesos.

2. Input gate: calculada com a finalidade de posteriormente atualizar o estado da célula atual $c_{t}$ (cell state). Aqui são pegos os valores de entrada $x_{t}$ e estado escondido $h_{t-1}$, e então aplicados na função de ativação recorrente $\sigma_{g}$ e numa segunda função, a tangente hiperbólica, denominada contextualmente como função de ativação $\sigma_{c}$. Ao final, é realizado o produto do resultado de ambas, onde a saída da ativação recorrente decide quanto de informação manter da saída da ativação;

$$
i_{t}=\sigma_{g}\left(W_{i} x_{t}+U_{i} h_{t-1}\right) \circ \sigma_{c}\left(W_{i} x_{t}+U_{i} h_{t-1}\right)
$$

3. Cell state: a partir deste ponto, o estado da célula atual é calculada. Primeiro, o estado da célula anterior é multiplicado pela saída do forget gate. Depois, os valores são somados com a saída do input gate;

$$
c_{t}=f_{t} \circ c_{t-1}+i_{t}
$$

4. Output gate: por último, aqui é calculado o estado oculto que será repassado para a próxima célula. O estado oculto anterior $h_{t-1}$ e a entrada atual $x_{t}$ são passados para a função de ativação recorrente $\sigma_{g}$, e sua saída é multiplicada pela saída da aplicação do novo estado da célula atual na função de ativação $\sigma_{c}$.

$$
o_{t}=\sigma_{g}\left(W_{o} x_{t}+U_{o} h_{t-1}\right) \circ \sigma_{c}\left(c_{t}\right)
$$


Outra variante bastante famosa é a GRU. Ela é uma ótima alternativa ao lidar com menor capacidade computacional, entretanto também tem menor capacidade representacional (CHOLLET, 2017). A variante segue o mesmo princípio da LSTM, porém é mais simples pois o fluxo de informação se divide em apenas duas partes, exigindo menos operações:

- Update gate: similar ao forget gate e o input gate do LSTM, decide quanto descartar ou manter da informação;

$$
z_{t}=\sigma_{g}\left(W_{z} x_{t}+U_{z} h_{t-1}\right)
$$

- Reset gate: decide quanto da informação do passado deve ser esquecida.

$$
r_{t}=\sigma_{g}\left(W_{r} x_{t}+U_{r} h_{t-1}\right)
$$

A saída do modelo GRU se dá pela seguinte equação:

$$
h_{t}=\left(1-z_{t}\right) \circ h_{t-1}+z_{t} \circ \sigma_{c}\left(W_{c} x_{t}+U_{c}\left(r_{t} \circ h_{t-1}\right)\right)
$$

A diferença estrutural entre as duas variantes pode ser observada na Figura 3, indicando as partes apontadas.

LSTM
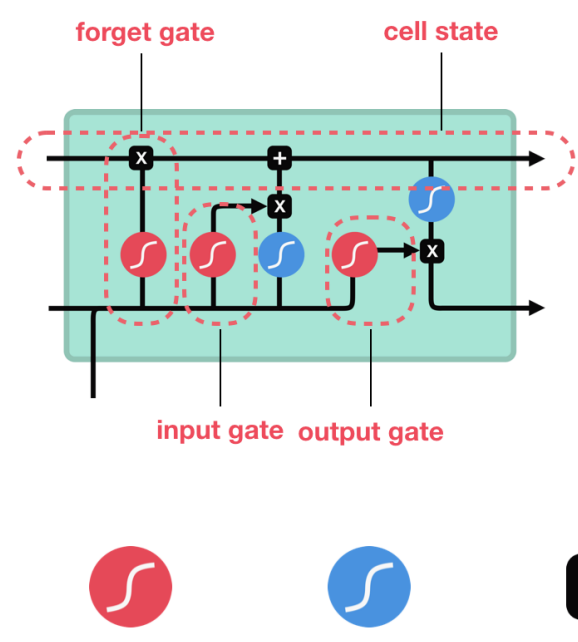

sigmoid

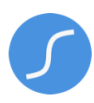

$\tanh$

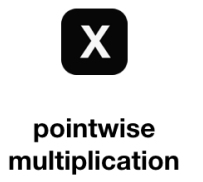

GRU

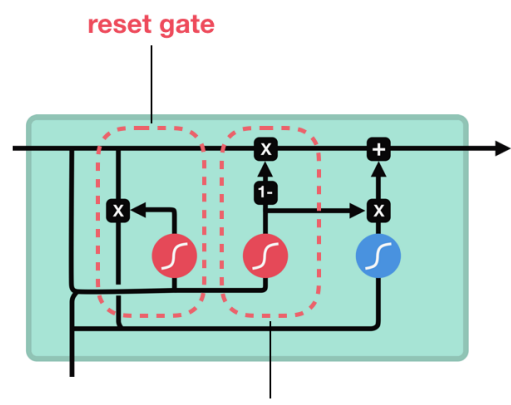

update gate

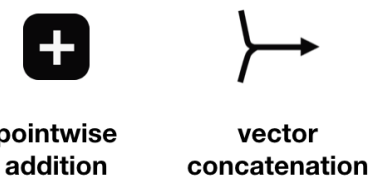

Figura 3 - Estrutura das células das unidades LSTM e GRU - Fonte: (Michael Nguyen, 2018) 


\subsubsection{Bidirecionamento de redes recorrentes}

Em problemas onde a ordem em que os dados foram registrados é significativa, RNNs executam bem o seu papel (CHOLLET, 2017). Entretanto, existem casos onde a ordem de captura de padrões necessita ser melhor explorada, não apenas em uma única direção. Goodfellow cita um exemplo em reconhecimento de fala:

"[...] a interpretação correta do som atual como um fonema pode depender dos próximos fonemas por causa da co-articulação, e potencialmente pode até depender das próximas palavras por causa das dependências linguísticas entre palavras próximas: se houver duas interpretações da palavra atual, que são ambas acusticamente plausíveis, talvez tenhamos que olhar para o futuro (e para o passado) para distingui-las." (GOODFELLOW et al., 2016, p.395)

Uma RNN bidirecional usa duas camadas de unidades, onde cada uma processa a sequência de entrada em direções diferentes (cronológica e anti-cronológica) e funde suas representações (CHOLLET, 2017). A Figura 4 mostra a computação de uma RNN bidirecional desenvolvida para mapear sequências de entrada $x$. A recorrência $h$ propaga a informação para frente no tempo (para a direita) enquanto a recorrência $g$ propaga a informação para trás no tempo (para a esquerda). Dessa forma, em cada passo de tempo $t$, as unidades de saída $\sigma(t)$ podem se beneficiar de conhecimento relevante do passado em sua entrada $h(t)$ e de conhecimento relevante do futuro em sua entrada $g(t)$ (GOODFELLOW et al., 2016).

\subsection{2 Árvores de decisão}

Árvores de decisão são estruturas similares a fluxogramas que permitem classificar pontos de dados de entrada ou valores de saída predeterminados, dadas as entradas (CHOLLET, 2017). Elas são consideradas fáceis de visualizar e interpretar, devido a simplicidade de suas estruturas. A Figura 5 apresenta uma estrutura genérica de uma árvore de decisão, na qual o dado de entrada passa através da árvore de acordo com as respostas (sim ou não) das perguntas que cada nó representa. Ao final, uma das categorias (ou classes) será a escolhida.

\subsubsection{Random Forest}

Random Forest é um classificador que consiste em uma coleção de árvores de decisão, onde cada árvore é construída aplicando um algoritmo no conjunto de treinamento e um vetor aleatório adicional, onde é amostrado independente e identicamente distribuída de alguma distribuição (SHALEV-SHWARTZ; BEN-DAVID, 2014). 


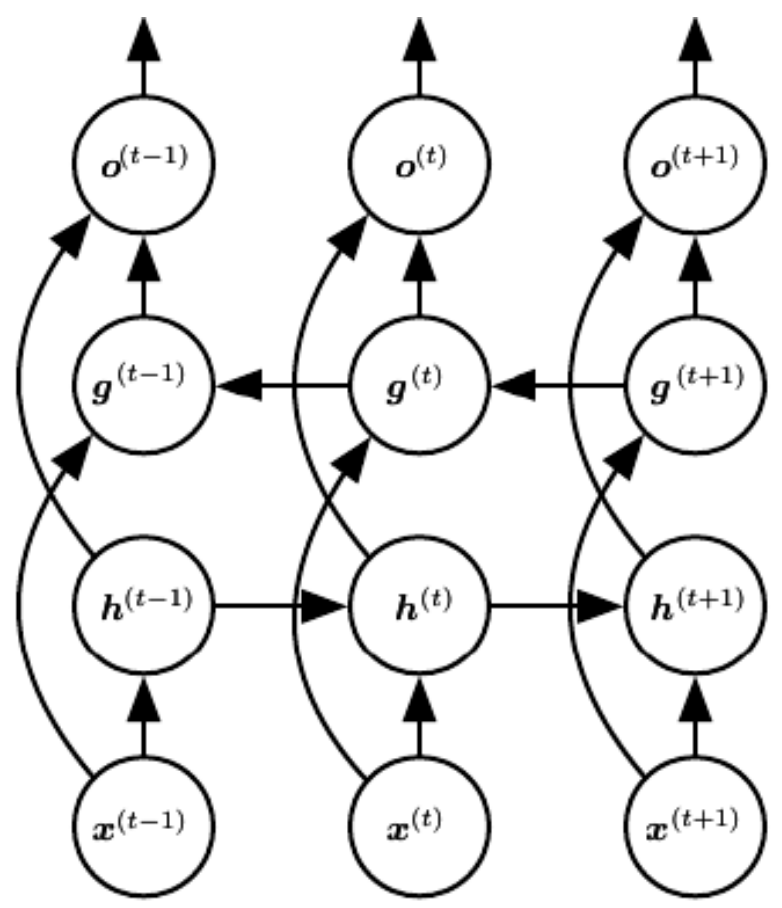

Figura 4 - Computação de uma RNN bidirecional - Fonte: (GOODFELLOW et al., 2016)

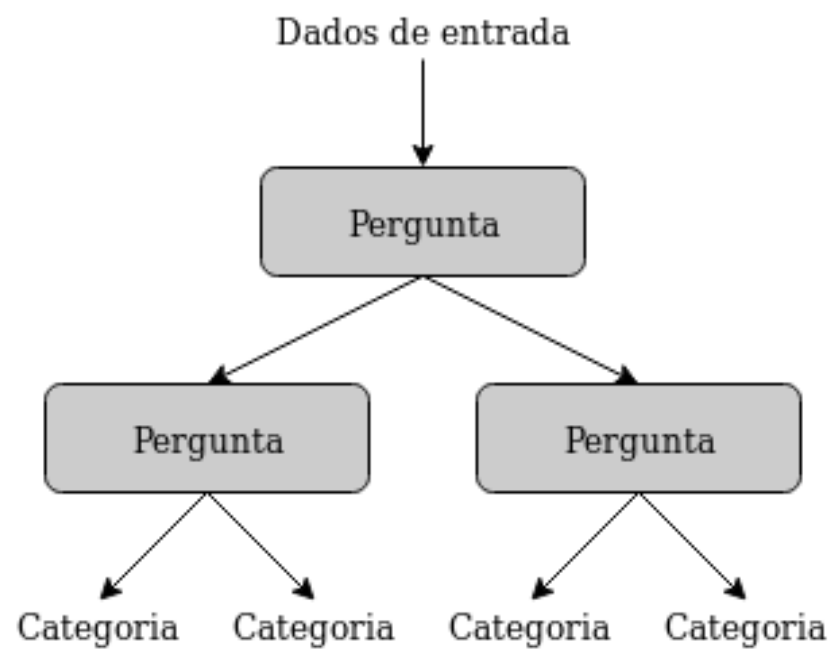

Figura 5 - Exemplo de estrutura de uma árvore de decisão

A predição de uma Random Forest é obtida por uma votação majoritária sobre as predições das árvores individuais. A Figura 6 traz uma representação simplista do conjunto de árvores de decisão que compõem uma Random Forest.

\subsubsection{Gradient Boost Machine}

Gradient Boost Machine é uma técnica de aprendizagem de máquina que se baseia em outros algoritmos de aprendizado de máquina, geralmente árvores de decisão, para produzir um modelo que permite o uso de um intensificador de gradiente 


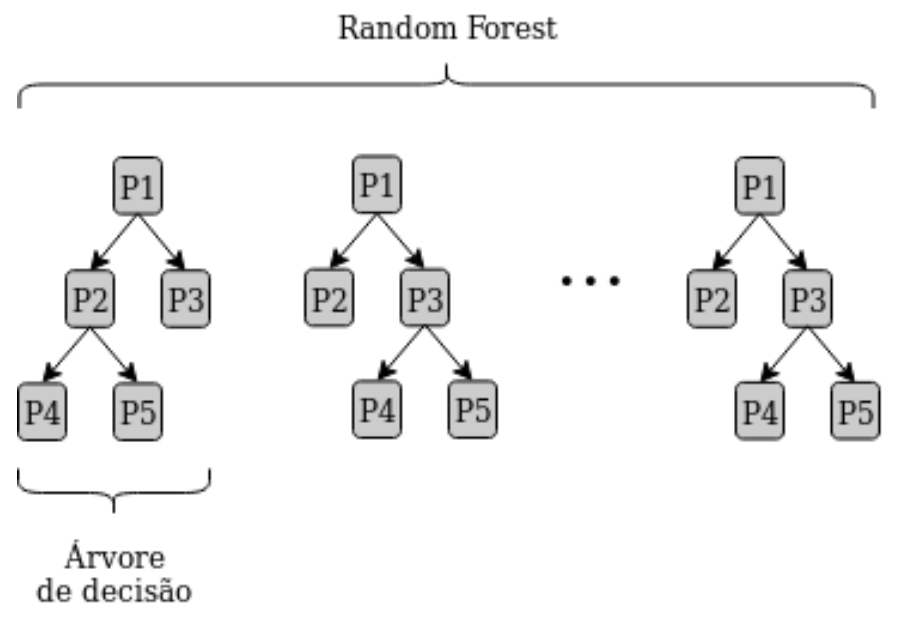

Figura 6 - Exemplo de conjunto de árvores de decisão de uma Random Forest

para otimizar o aprendizado do modelo (CHOLLET, 2017). Ou seja: a medida que o classificador é treinado, o ruído é calculado e o modelo é então melhorado.

\subsubsection{Estratégia One-vs-the-rest (OvR)}

A estratégia OvR consiste em configurar um classificador binário por classe, onde para cada classificador as amostras da classe são ajustadas como positivas e o restante das outras classes como negativas (BISHOP, 2006).

Um exemplo é a classificação entre 3 classes: A, B e C. Se aplicado OvR, o classificador será composto por 3 estimadores binários (um por classe), onde cada um visualizará as classes de maneiras distintas. No estimador 1, para a classe A a saída deverá ser 1 (verdadeiro), enquanto que para as classes $\mathrm{B}$ e $\mathrm{C}$ a saída deverá ser 0 (falso). Já o estimador 2 para classe $B$ a saída deverá ser 1 , e para restante das classes 0 . $O$ estimador 3 para classe $C$ deverá ser 1 , e o restante das classes 0 .

Segundo (SHALEV-SHWARTZ; BEN-DAVID, 2014), esta é a abordagem mais simples para lidar com problemas de predição para múltiplas classes.

\subsection{Trabalhos relacionados}

O RAH atraiu atenção da pesquisa em diversos âmbitos, principalmente por sua capacidade de extrair importantes conhecimentos sobre a atividade humana a partir de dados brutos de sensores (WANG et al., 2019). A seguir são apresentados alguns dos trabalhos de pesquisa dedicados a $\mathrm{RAH}$, que utilizaram modelos de aprendizagem de máquina para resolver problemas dentro de áreas específicas.

(LI et al., 2017) adotou um modelo CNN para detectar quedas através de dados de vídeo, para aprender características de deformação da forma humana que possam 
descrever diferentes posturas humanas e determinar se a queda realmente ocorreu. Sua abordagem alcançou uma acurácia média de 99,98\% nos experimentos utilizando validação cruzada.

(YANG et al., 2015) também desenvolveu um classificador CNN para RAH, porém para alimentá-lo com um tipo diferente de dado: dados inerciais de um conjunto de sensores vestíveis. Ela obteve médias de acurácia de 86,8\% e 95\% para duas bases de dados específicas: Opportunity Activity Recognition ${ }^{1}$ e Hand Gesture ${ }^{2}$.

(SUN et al., 2019) propôs um sistema preliminar de classificação de comportamento de jogadores de beisebol, através de um modelo LSTM e dados de sensores e câmeras. Os autores apontam três contribuições: 1 . Sinais de uma câmera de profundidade e de múltiplos sensores inerciais são obtidos e segmentados; 2. A projeção vetorial de esqueleto variante no tempo da câmera de profundidade e features estatísticas extraídas dos sensores inerciais são usados como features do modelo; e 3. Um esquema baseado em deep learning é proposto para o treinamento de classificadores de comportamento. Os resultados experimentais demonstraram que o sistema proposto atinge acurácia maior que $95 \%$.

Modelos LSTM foram aplicados em outros trabalhos de pesquisa também, como (CHUNG et al., 2019) para detecção atividades diárias (comer, dirigir, etc), e (NEVEROVA et al., 2016) para identificar humanos individualmente através de padrões de movimento do smartphone, ambos com taxa acurácia por volta dos 93\%.

Diferente dos modelos citados anteriormente, (DING et al., 2018) propôs um modelo CNN + LSTM. O objetivo era detectar comportamentos perigosos em canteiros de obras. Segundo ele, a extração de conhecimento neste contexto representou um grande desafio devido o grau de complexidade das features espaço-temporais, iluminação e o conjunto de pontos de vista necessários para identificar uma ação insegura. O modelo híbrido de deep learning proposto conseguiu $97 \%$ de acurácia e foi divido em quatro etapas:

1. Identificar ações inseguras;

2. Coletar dados de movimento e vídeos do local;

3. Extrair os recursos visuais dos vídeos usando um modelo CNN;

4. Sequenciar as features ativadas pelo uso do modelo LSTM.

1 "UCl Machine Learning Repository: OPPORTUNITY Activity Recognition Data Set" https://archive.ics.uci.edu/ml/datasets/opportunity+activity+recognition. Acessado em 14 jul. 2019.

2 "GitHub - andreas-bulling/ActRecTut: MATLAB Human Activity Recognition Toolbox" https://github.com/andreas-bulling/ActRecTut. Acessado em 14 jul. 2019. 
Outros trabalhos também desenvolveram modelos CNN + LSTM para RAH, como (NÚÑEZ et al., 2018) para reconhecer atividades e gestos de dados 3D de esqueletos (até $99 \%$ de acurácia entre as bases de dados consideradas), ou (ORDÓÑEZ; ROGGEN, 2016) para identificar tarefas simples como abrir e fechar portas, limpar uma mesa ou mexer num interruptor (92\% de acurácia para a base de dados Opportunity Activity Recognition e 94\% de acurácia para a base de dados SKODA).

Diferentemente dos trabalhos aqui citados nesta seção, este trabalho de conclusão de curso busca elencar e avaliar não apenas classificadores de deep learning recorrentes, mas também bidirecionais, além de compará-los com outros algoritmos de aprendizagem de máquina tradicionais, baseados em árvores de decisão. Além disso, as atividades que os modelos atenderam são mais diversificadas, não atreladas a tarefas ou contextos específicos. 


\section{Modelos para detecção de atividades humanas}

Uma vez que os modelos são fortemente dependentes dos dados de entrada e dos cenários a serem classificados, primeiramente, este capítulo apresenta a base de dados, os cenários e as atividades humanas que serão consideradas pelos modelos propostos (Seção 3.1), assim como os modelos classificadores desenvolvidos para detectar estas atividades e as ferramentas utilizadas para construção destes modelos (Seção 3.2).

\subsection{Cenários e data set}

A UP-Fall Detection é uma base de dados disponibilizada pela organização do Challenge UP ${ }^{8}$, uma competição organizada pela Universidad Panamericana para o fomento da comunidade científica voltado para reconhecimento de atividade humana e detecção de quedas (CHALLENGE..., 2019).

Os dados da UP-Fall Detection foram gerados numa abordagem multimodal, através dos dispositivos listados a seguir:

- Cinco sensores vestíveis Mbientlab MetaSensor, dispondo dados de aceleração e velocidade angular em três eixos, além de luminosidade;

- Um headset eletroencefalógrafo (EEG) NeuroSky MindWave, configurado para mensurar apenas o sinal de onda do canal EEG localizado na testa do usuário;

- Seis sensores infravermelhos localizados acima do piso da sala, realizando as medições das mudanças nas interrupções dos dispositivos ópticos;

- Duas câmeras Microsoft LifeCam Cinema, uma para visão lateral e outra para visão frontal da sala.

De maneira sucinta, (JEWITT, 2009) explica que a multimodalidade é uma abordagem de representação, comunicação e interação que observa além da linguagem para investigar a multiplicidade de formas como nos comunicamos: por meio de imagens, sons e músicas, gestos, postura corporal e uso do espaço. Estas múltiplas

8 "Challenge UP." https://sites.google.com/up.edu.mx/challenge-up-2019/. Acessado em 4 jun. 2019. 
vias de transmissão de informação podem ser representadas em forma de dados brutos, através de sensores e outros tipos dispositivos de captura, como câmeras e gravadores de som.

A Figura 7 apresenta como os dispositivos foram posicionados nos experimentos de coleta de dados; sensores vestíveis e headset EEG no corpo humano e sensores infravermelhos e câmeras no ambiente de experimento. O Anexo A apresenta a lista de todos os dispositivos utilizados e seus respectivos dados coletados.

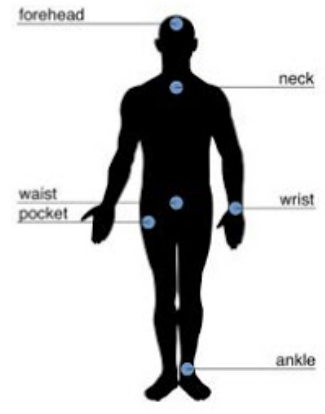

(a)

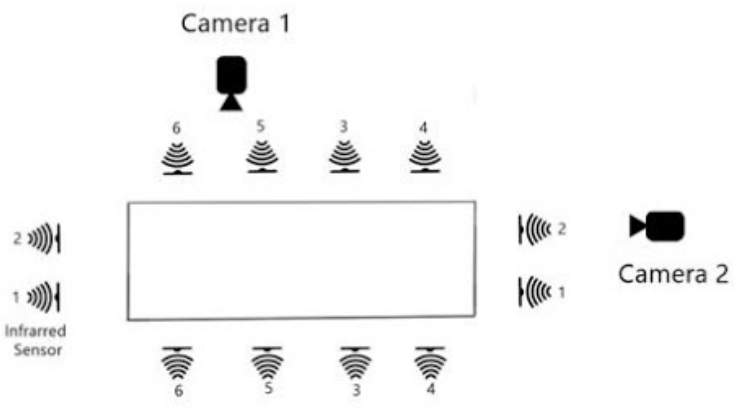

(b)

Figura 7 - Distribuição dos sensores usados para coletar os dados: (a) Sensores vestíveis e headset EEG localizados no corpo humano, e (b) Layout dos sensores infravermelho e câmeras - Fonte: (CHALLENGE. .., 2019).

As atividades que os modelos propostos neste trabalho serão capazes de classificar estão enumeradas e descritas na Tabela 1. Parte destas atividades tem como foco diferentes tipos de queda (atividades de 1 a 5), seguidas por atividades mais comuns do cotidiano (atividades 6 a 12).

Tabela 1 - Atividades e suas respectivas descrições

\begin{tabular}{|c|c|}
\hline Atividade & Descrição \\
\hline 1 & Caindo para frente com as mãos \\
\hline 2 & Caindo para frente com os joelhos \\
\hline 3 & Caindo para trás \\
\hline 4 & Caindo de lado \\
\hline 5 & Caindo ao sentar \\
\hline 6 & Andando \\
\hline 7 & Parado em pé \\
\hline 8 & Sentado \\
\hline 9 & Pegando um objeto \\
\hline 10 & Pulando \\
\hline 11 & Deitado \\
\hline 12 & Ajoelhado \\
\hline
\end{tabular}

Estas atividades foram registradas em sequências de estados referentes ao comportamento do corpo no decorrer do tempo, podendo apresentar padrões como mostra a Figura 8. 


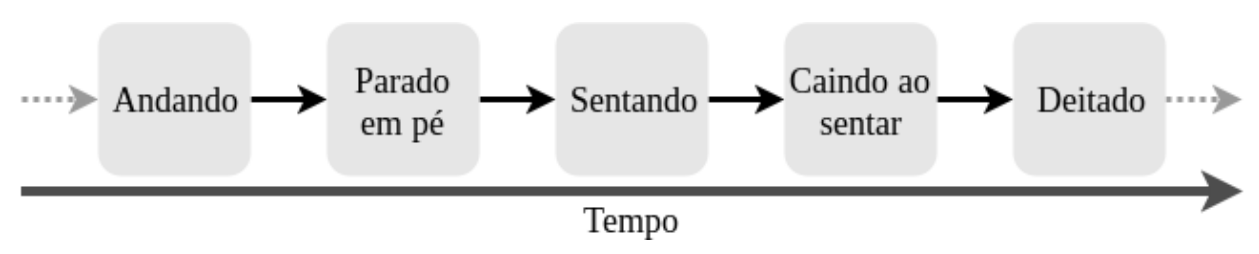

Figura 8 - Exemplo de sequência de atividades no tempo

As atividades foram realizadas por 9 pessoas com idades entre 18 e 24 anos, que possuem diferentes características físicas. Cada pessoa dedicou 3 tentativas para cada uma das atividades que realizou, com tempos de duração que variaram entre 10 e 60 segundos. Ao total, a base é composta por 160.736 registros.

\subsection{Modelos}

A seguir são apresentados quatro modelos de aprendizado de máquina, desenvolvidos para realizar a classificação das 12 (doze) atividades descritas na Seção 3.1. O número de canais em que são disponibilizados os valores numéricos dos sensores foi ajustado com o objetivo de obter o desempenho máximo dos classificadores utilizando a menor quantidade de sensores possível.

Durante o processo de seleção dos canais, percebeu-se que os dados dos sensores de luminosidade fizeram o desempenho cair muito para os modelos neurais. Já para os modelos baseados em árvore de decisão, estes foram essenciais (quase que suficientes) para o bom desempenho.

De forma empírica, 24 dos 44 canais disponíveis foram selecionados para uso no processo de treinamento do modelos neurais (Tabela 2), enquanto que para os modelos baseados em árvore de decisão foram selecionados apenas 11 (Tabela 3).

\subsubsection{LSTM Bidirecional}

A Figura 9 apresenta o modelo LSTM Bidirecional proposto neste trabalho para detectar as atividades descritas anteriormente. O modelo é estruturado em duas camadas bidirecionais com 200 (duzentas) unidades LSTM cada. As funções de ativação e ativação recorrente setadas são tangente hiperbólica e sigmóide, respectivamente. A entrada do modelo recebe blocos de dados em sequência, onde cada bloco representa uma amostra de 1 segundo. A saída do modelo é uma camada totalmente conectada com 12 (doze) unidades e função de ativação softmax.

Para função de perda é a categorical cross-entropy, uma vez que é ótima ao lidar com classificação de múltiplos rótulos (ZHAO et al., 2016). Para otimizador, o modelo utiliza o algoritmo Adam (KINGMA; BA, 2014) configurado com os seguintes 
Tabela 2 - Canais selecionados para o treinamento dos modelos neurais

\begin{tabular}{|c|c|}
\hline Local do dispositivo & Canal \\
\hline \multirow{4}{*}{ Tornozelo } & Acelerômetro (eixo x) \\
& Acelerômetro (eixo y) \\
& Acelerômetro (eixo z) \\
& Giroscópio (eixo x) \\
& Giroscópio (eixo y) \\
& Giroscópio (eixo z) \\
\hline \multirow{5}{*}{ Bolso direito } & Acelerômetro (eixo x) \\
& Acelerômetro (eixo y) \\
& Acelerômetro (eixo z) \\
& Giroscópio (eixo x) \\
& Giroscópio (eixo y) \\
& Giroscópio (eixo z) \\
\hline \multirow{5}{*}{ Cintura } & Acelerômetro (eixo x) \\
& Acelerômetro (eixo y) \\
& Acelerômetro (eixo z) \\
& Giroscópio (eixo x) \\
& Giroscópio (eixo y) \\
& Giroscópio (eixo z) \\
\hline Pescoço & Acelerômetro (eixo x) \\
& Acelerômetro (eixo y) \\
& Acelerômetro (eixo z) \\
& Giroscópio (eixo x) \\
& Giroscópio (eixo y) \\
& Giroscópio (eixo z)
\end{tabular}

Tabela 3 - Canais selecionados para o treinamento dos modelos baseados em árvore de decisão

\begin{tabular}{|c|c|}
\hline Local do dispositivo & Canal \\
\hline Tornozelo & $\begin{array}{l}\text { Acelerômetro (eixo x) } \\
\text { Acelerômetro (eixo z) } \\
\text { Luminosidade }\end{array}$ \\
\hline Bolso direito & $\begin{array}{l}\text { Acelerômetro (eixo x) } \\
\text { Acelerômetro (eixo z) } \\
\text { Luminosidade }\end{array}$ \\
\hline Cintura & Luminosidade \\
\hline Pescoço & $\begin{array}{c}\text { Acelerômetro (eixo x) } \\
\text { Acelerômetro (eixo z) } \\
\text { Luminosidade }\end{array}$ \\
\hline Pulso & Luminosidade \\
\hline
\end{tabular}

parâmetros: taxa de aprendizagem $=0.001, \beta_{1}=0.9$ e $\beta_{2}=0.999$. Vale ressaltar que todos os parâmetros do modelo foram configurados empiricamente.

Por fim o treinamento do modelo consistiu em 30 épocas, alcançando o máximo de seu desempenho. Após estas épocas, o modelo tende a manter resultados muito próximos entre épocas em relação as métricas de performance. Na Figura 10 pode-se observar a taxa de acurácia do modelo no decorrer das épocas de treinamento. 


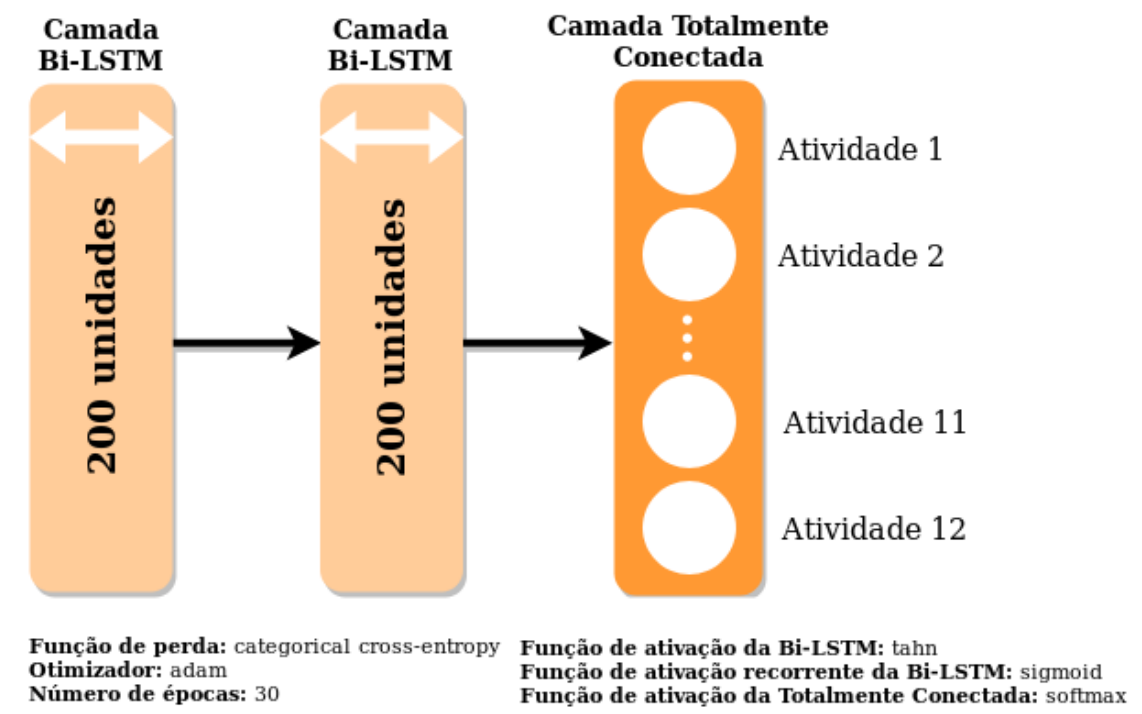

Figura 9 - Modelo LSTM Bidirecional

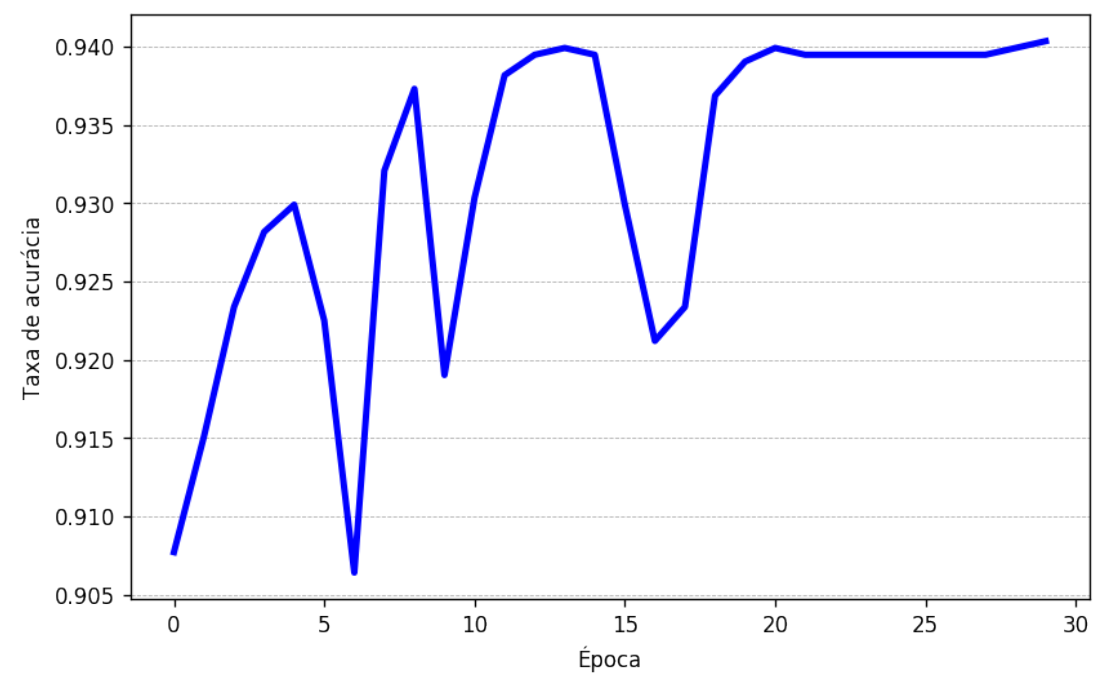

Figura 10 - Acurácia do modelo LSTM Bidirecional através das épocas

\subsubsection{GRU Bidirecional}

O modelo GRU Bidirecional (apresentado na Figura 11) segue uma topologia similar ao modelo LSTM Bidirecional descrito anteriormente, utilizando duas camadas bidirecionais e 200 unidades em cada. A entrada do modelo GRU Bidirecional também recebe dados sequenciados no formato de blocos, e cada bloco representa uma amostra de 1 segundo.

A tangente hiperbólica continua como função de ativação destas unidades, porém a função de ativação recorrente é a hard sigmoid. A configuração da saída, da função de perda e do otimizador são os mesmos do modelo LSTM bidirecional. $\mathrm{O}$ treinamento também utilizou 30 épocas, para alcançar os melhores resultados. A 


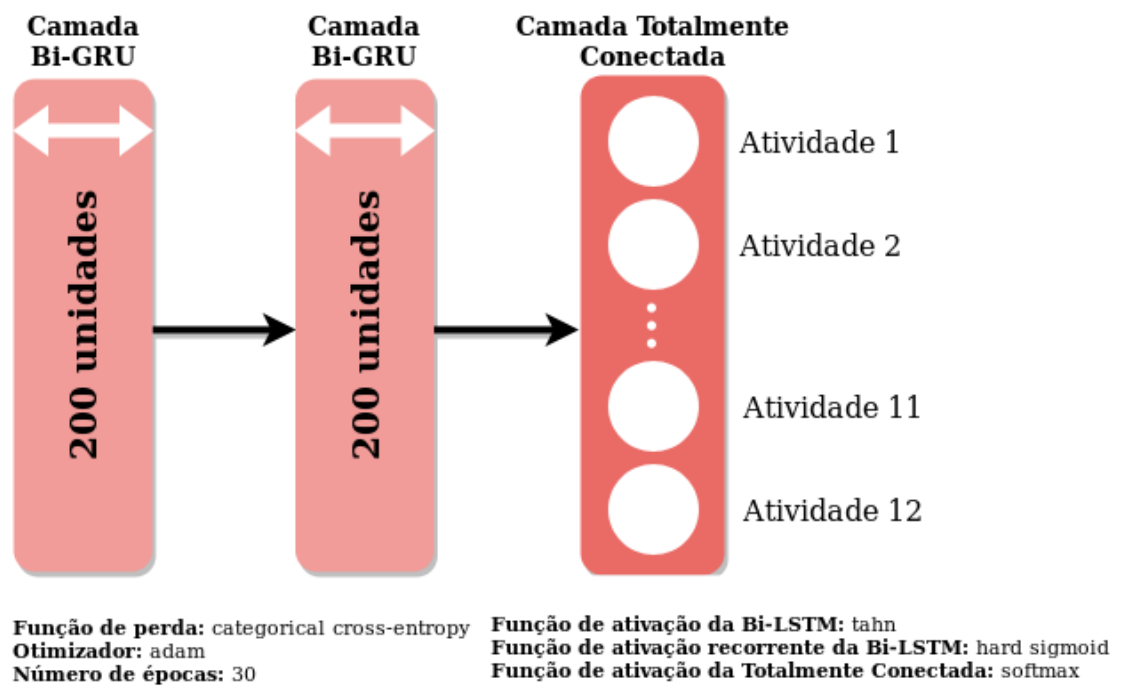

Figura 11 - Modelo GRU Bidirecional

Figura 12 apresenta a taxa de acurácia do modelo no decorrer das épocas de treinamento.

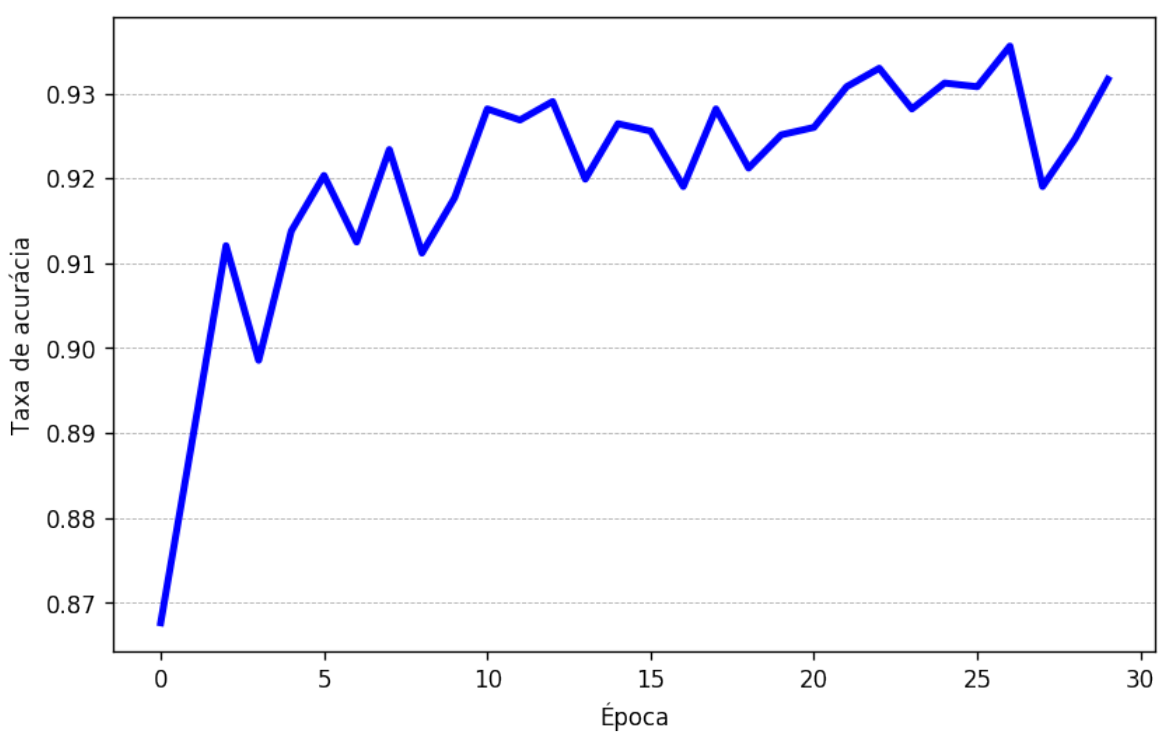

Figura 12 - Acurácia do modelo GRU Bidirecional através das épocas

\subsubsection{Random Forest com OvR}

A Figura 13 apresenta o modelo Random Forest com OvR proposto neste trabalho. Os estimadores são compostos por 15 árvores de decisão com profundidade máxima de 36 cada, e replicados um para cada rótulo (12 Random Forests binárias). A entrada do modelo recebe dados linha a linha. 


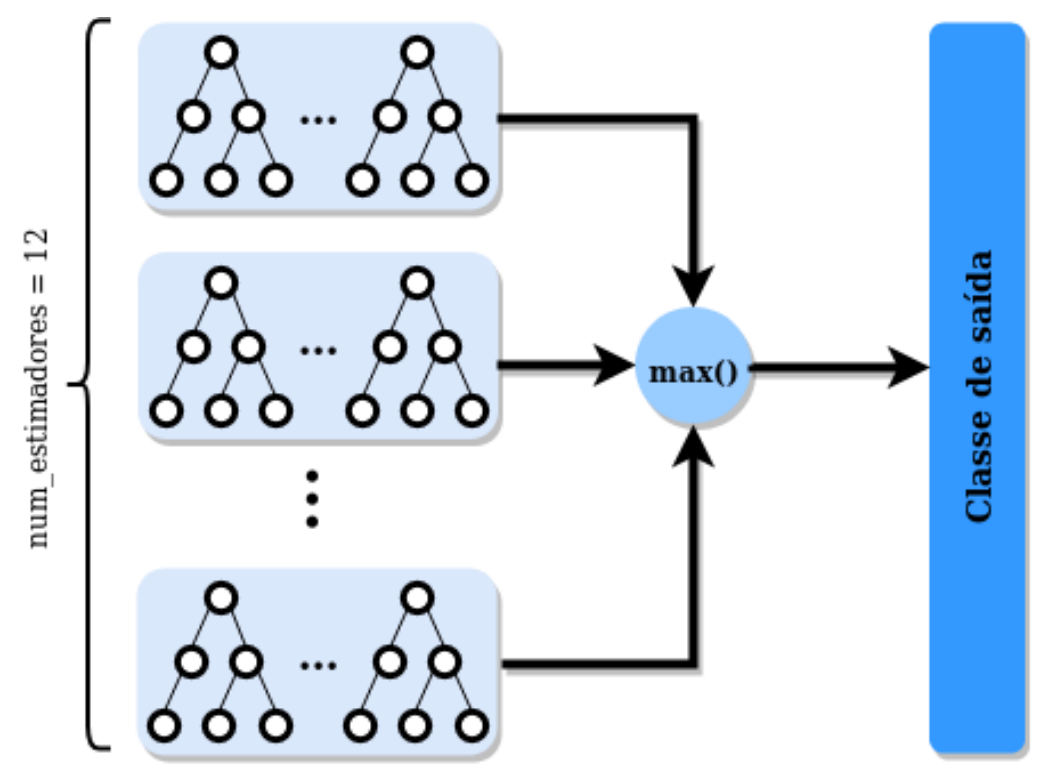

Número de árvores por estimador: 15 Profundidade máxima da árvore: 36

Figura 13 - Modelo Random Forest com estratégia OvR

Outros parâmetros do modelo foram o mínimo de duas amostras para dividir um nó e mínimo de uma amostra para criar um nó folha. Todas as configurações do modelo foram adquiridas empiricamente.

\subsubsection{Gradient Boost Machine com OvR}

A Figura 14 apresenta o modelo Gradient Boost Machine com OvR. A topologia deste último classificador é similar ao Random Forest pois utiliza 12 classificadores binários compostos por 15 árvores de decisão de profundidade máxima 36 . A entrada do modelo também recebe dados linha a linha.

A função objetiva destes classificadores é a logística, taxa de aprendizado $=$ 0.9 e pontuação base $=0.5$. Todas as configurações do modelo foram adquiridas empiricamente.

\subsubsection{Implementação dos modelos}

Todos os modelos propostos neste trabalho foram implementados no ambiente do Google Colaboratory ${ }^{1}$, utilizando a linguagem Python ${ }^{2}$ versão 3.6 e os seguintes frameworks:

\footnotetext{
1 "Google Colaboratory." https://colab.research.google.com/. Acessado em 4 jun. 2019.

2 "Python.org." https://www.python.org/. Acessado em 4 jun. 2019.
} 


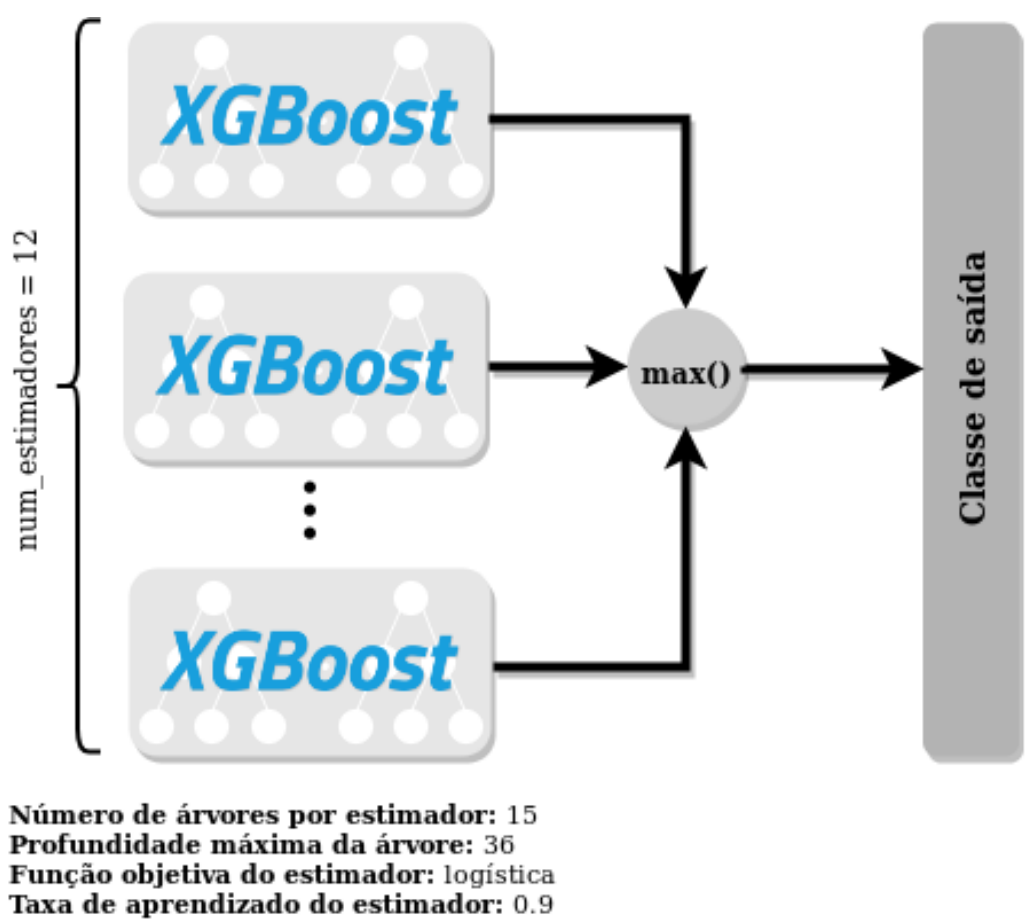

Figura 14 - Modelo Gradient Boost Machine com estratégia OvR

- NumPy ${ }^{3}$ : suporta arrays e matrizes multidimensionais, possuindo grande variedade de funções matemáticas para trabalhar com estas estruturas;

- Pandas ${ }^{4}$ : biblioteca de código aberto, licenciada BSD, que fornece estruturas de dados rápidas, flexíveis e expressivas, projetadas para trabalhar com dados relacionais ou rotulados de maneira fácil e intuitiva;

- Tensorflow ${ }^{5}$ : plataforma de código aberto com ecossistema de ferramentas, bibliotecas e recursos da comunidade para desenvolvimento de modelos de aprendizagem de máquina no estado da arte;

- Keras ${ }^{6}:$ API de redes neurais de alto nível escrita em Python, com foco em permitir rápidos experimentos. Traz suporte ao Theano e Tensorflow como backend;

- Scikit-learn ${ }^{7}$ : biblioteca de aprendizado de máquina de código aberto que inclui vários algoritmos de classificação, regressão e agrupamento; e

- XGBoost ${ }^{8}$ : biblioteca de otimização de gradiente distribuída, que implementa algoritmos de aprendizado de máquina sob a estrutura Gradient Boosting.

\footnotetext{
"NumPy - NumPy." https://www.numpy.org/. Acessado em 4 jun. 2019.

"Pandas." https://pandas.pydata.org/. Acessado em 4 jun. 2019.

"Tensorflow." https://www.tensorflow.org/. Acessado em 13 jun. 2019.

"Keras Documentation: Home." http://keras.io/. Acessado em 4 jun. 2019.

"Scikit-learn." https://scikit-learn.org/. Acessado em 4 jun. 2019.

"XGBoost Documentation.." https://xgboost.readthedocs.io/en/latest/. Acessado em 4 jun. 2019.
} 
A configuração da máquina virtual disponibilizada pelo ambiente consiste em 12GB de memória RAM, 350GB de armazenamento em disco, processador Intel Xeon CPU 2.30GHz e GPU Tesla T4 com 15.8GB de memória dedicada. 


\section{Resultados}

Neste capítulo serão apresentadas as métricas utilizadas para avaliar os modelos (Seção 4.1), comparações de desempenho entre os modelos (Seção 4.2), e ao final uma discussão geral a respeito dos resultados (Seção 4.3).

\subsection{Métricas para avaliação de desempenho}

Para avaliar o desempenho dos modelos foram selecionadas cinco métricas, calculadas com base nos verdadeiros positivos (VP), verdadeiros negativos (VN), falsos positivos (FP) e falsos negativos (FN) das predições. São estas:

- Acurácia: taxa entre número total de acertos e número total de exemplos.

$$
\text { Acurácia }=\frac{V \text { Ptotal }+V N_{\text {total }}}{V P_{\text {total }}+F P_{\text {total }}+F N_{\text {total }}+V N_{\text {total }}}
$$

Esta métrica expressa uma noção geral do desempenho do modelo. Ela não leva em consideração o eventual desbalanceamento da quantidade de exemplos para cada classe.

- Precisão: taxa entre verdadeiros positivos e todos os exemplos positivos previstos de uma classe.

$$
\text { Precisão }=\frac{V P_{\text {classe }}}{V P_{\text {classe }}+F P_{\text {classe }}}
$$

Mensura quanto dos exemplos previstos para serem classificados por uma classe foram de fato rotulados.

- Recall ou sensibilidade: taxa de verdadeiros positivos para cada exemplo rotulado por uma classe.

$$
\text { Recall }=\frac{V P_{\text {classe }}}{V P_{\text {classe }}+F N_{\text {classe }}}
$$

Mensura quanto dos exemplos rotulados por uma classe realmente eram desta classe.

- Especificidade: taxa entre verdadeiros negativos e todos os exemplos não rotulados por uma classe. 


$$
\text { Especificidade }=\frac{V N_{\text {classe }}}{V N_{\text {classe }}+F P_{\text {classe }}}
$$

Mensura quanto dos exemplos não rotulados por uma classe foram corretamente não rotulados.

- F1-score: média ponderada da precisão com a recall.

$$
F 1=2 \times \frac{\text { Precisão } \times \text { Recall }}{\text { Precisão }+ \text { Recall }}
$$

Esta métrica pode ser calculada tanto para uma classe específica quanto para o modelo como um todo. Neste último caso, utiliza-se a média das precisões e recalls de todas as classes como parâmetros da função. Ao contrário da acurácia, a F1-score consegue contemplar uma noção de desempenho para cada uma das classes, mesmo com a base de dados desbalanceada.

\subsection{Comparações e análises}

Para treino e teste dos modelos, a base de dados descrita na Seção 3.1 foi dividida em $75 \%$ e $25 \%$, respectivamente. A seguir na Subseção 4.2 .1 são apresentadas as matrizes de confusão geradas durante os testes dos modelos. A Subseção 4.2.2 aborda os resultados das métricas.

\subsubsection{Matriz de confusão}

A Figura 15 representa a matriz de confusão referente ao modelo LSTM Bidirecional. O modelo apresenta maior quantidade de VP para as classes $6,7,8,10$ e 11. Há diversas inconsistências no processo de rotulação das atividades, como a atividade 3 (caindo para trás) erroneamente rotulada como 5 (caindo ao sentar), e a atividade 7 (parado em pé) erroneamente rotulada como 9 (pegando um objeto).

Na Figura 16 apresenta a matriz de confusão do modelo GRU Bidirecional. O padrão de quantidades apresentado pelo modelo LSTM Bidirecional se mantém para o modelo GRU Bidirecional, indicando alta quantidade de VP nas classes 6, 7, 8, 10 e 11. Também mantém inconsistências de rotulação como ocorre com as atividades de 8 a 11, que são erroneamente rotuladas como atividade 7.

Esses erros por parte dos modelos de Deep Learning se devem ao fato que tal classe de redes neurais extrai padrões de grande quantidades de dados. Uma vez que há um desbalanceamento muito grande, com classes que tem quase 65 vezes mais dados de treinamento que outras (por exemplo, a classe 2 tem 531 exemplos e a classe 11 tem 34.299 exemplos), o modelo acaba generalizando de forma incorreta. Assim, 


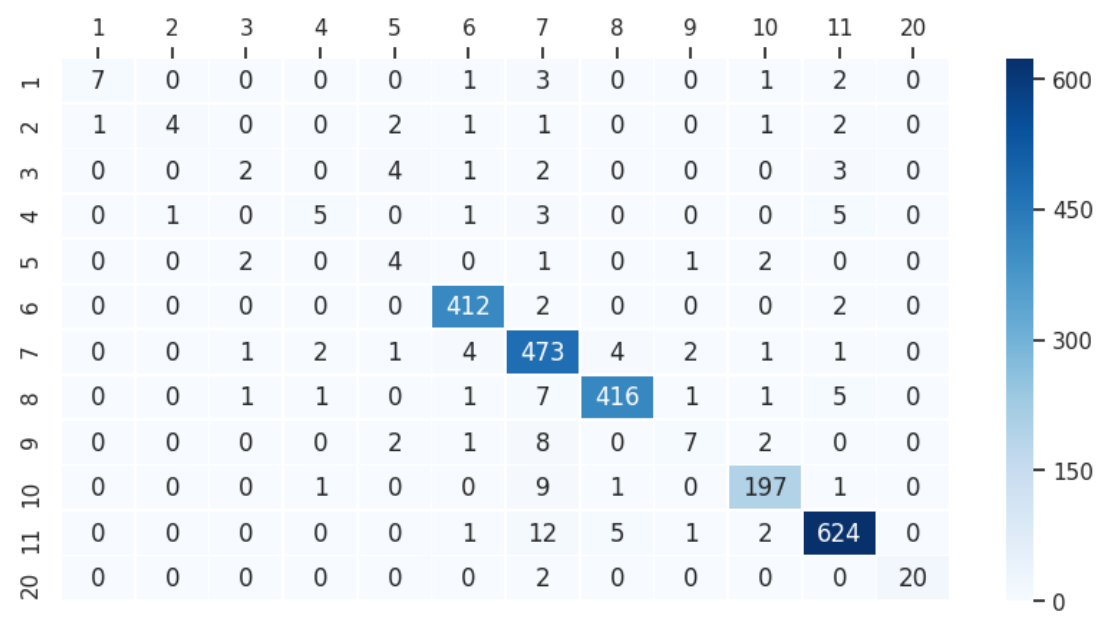

Figura 15 - Matriz de confusão - LSTM Bidirecional

\begin{tabular}{|c|c|c|c|c|c|c|c|c|c|c|c|c|c|}
\hline & 1 & 2 & 3 & 4 & 5 & 6 & 7 & 8 & 9 & 10 & 11 & 20 & \\
\hline$\rightarrow$ & $\begin{array}{l}1 \\
3\end{array}$ & $\begin{array}{l}1 \\
3\end{array}$ & $\begin{array}{l}1 \\
0\end{array}$ & $\begin{array}{l}1 \\
0\end{array}$ & $\begin{array}{l}\text { ' } \\
\text { on }\end{array}$ & $\begin{array}{l}1 \\
1\end{array}$ & $\begin{array}{l}1 \\
3\end{array}$ & $\begin{array}{l}1 \\
0\end{array}$ & $\begin{array}{l}1 \\
0\end{array}$ & $\begin{array}{l}1 \\
3\end{array}$ & $\begin{array}{l}1 \\
1\end{array}$ & $\begin{array}{l}1 \\
0\end{array}$ & -600 \\
\hline N & 0 & 5 & 0 & 0 & 2 & 0 & 1 & 0 & 0 & 3 & 1 & 0 & \\
\hline$m$ & 0 & 2 & 2 & 1 & 1 & 0 & 1 & 1 & 0 & 2 & 2 & 0 & \\
\hline$\sigma$ & 0 & 2 & 0 & 6 & 0 & 2 & 1 & 0 & 0 & 0 & 4 & 0 & -450 \\
\hline in & 0 & 0 & 1 & 0 & 4 & 0 & 4 & 0 & 0 & 1 & 0 & 0 & \\
\hline 6 & 2 & 0 & 0 & 0 & 0 & 411 & 2 & 0 & 0 & 1 & 0 & 0 & \\
\hline$r$ & 1 & 1 & 1 & 0 & 0 & 9 & 470 & 3 & 1 & 1 & 2 & 0 & 300 \\
\hline$\infty$ & 0 & 2 & 0 & 0 & 2 & 0 & 17 & 406 & 0 & 2 & 4 & 0 & \\
\hline$a$ & 0 & 1 & 0 & 0 & 1 & 0 & 11 & 1 & 4 & 0 & 1 & 1 & \\
\hline 윽 & 0 & 0 & 0 & 0 & 0 & 0 & 9 & 1 & 0 & 198 & 1 & 0 & \\
\hline$\exists$ & 1 & 1 & 0 & 1 & 2 & 2 & 9 & 2 & 0 & 3 & 624 & 0 & \\
\hline ำ & 0 & 0 & 0 & 0 & 0 & 0 & 1 & 0 & 0 & 0 & 0 & 21 & \\
\hline
\end{tabular}

Figura 16 - Matriz de confusão - GRU Bidirecional

é plausível que classes que tenham menos dados que outras sejam classificadas de forma errada.

A matriz de confusão do modelo Random Forest com OvR é apresentada na Figura 17. Neste caso, as classes 6, 7, 8, 10 e 11 continuam com uma quantidade alta de VP, porém percebe-se que o erro de classificação das outras classes diminuiu consideravelmente. Vale destacar as atividades de 1 a 5 (caindo) erroneamente rotuladas como 11 (deitado), atividade 2 (caindo para frente com os joelhos) erroneamente rotulada como 7 (parado em pé), e atividade 7 erroneamente rotulada como 9 (pegando um objeto).

Por fim, a Figura 18 apresenta a matriz de confusão para o Gradient Boost Machine com OvR. Em relação ao Random Forest com OvR, VP aumenta para as classes de 1 a 5,7 e 9 em até 8,62\%, mantiveram para a classe 8 e pioraram em até $1 \%$ para as outras classes. 


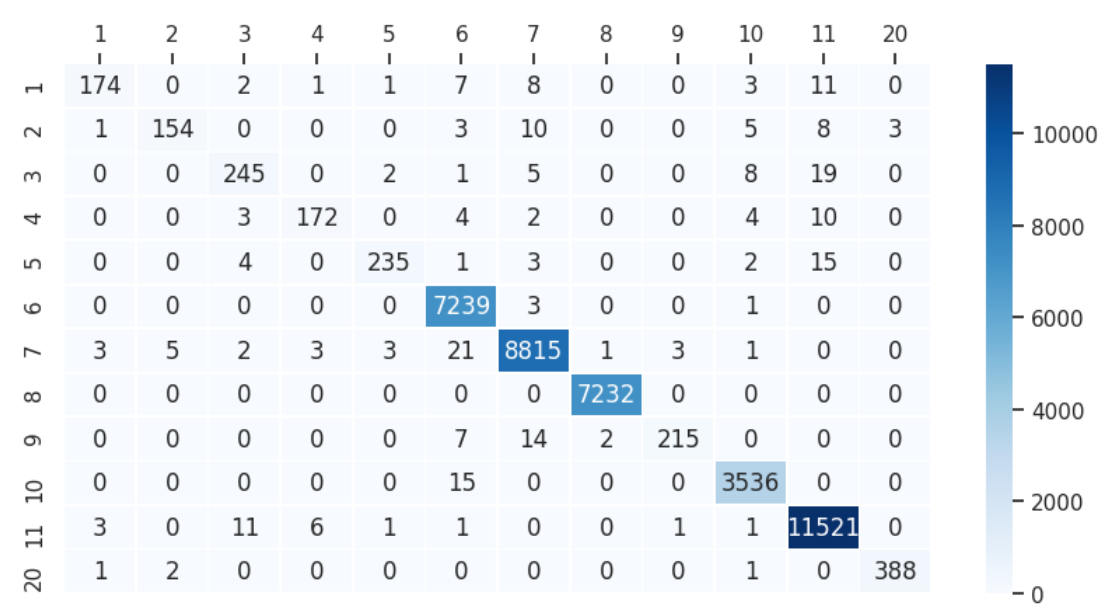

Figura 17 - Matriz de confusão - Random Forest com OvR

\begin{tabular}{|c|c|c|c|c|c|c|c|c|c|c|c|c|c|}
\hline & $\begin{array}{l}1 \\
1\end{array}$ & $\begin{array}{l}2 \\
1\end{array}$ & $\begin{array}{l}3 \\
1\end{array}$ & $\begin{array}{l}4 \\
1\end{array}$ & $\begin{array}{l}5 \\
1\end{array}$ & $\begin{array}{l}6 \\
1\end{array}$ & $\begin{array}{l}7 \\
1\end{array}$ & $\begin{array}{l}8 \\
1\end{array}$ & $\begin{array}{l}9 \\
1\end{array}$ & $\begin{array}{c}10 \\
1\end{array}$ & $\begin{array}{c}11 \\
1\end{array}$ & $\begin{array}{c}20 \\
1\end{array}$ & \multirow{4}{*}{ - 10000} \\
\hline- & 189 & 0 & 0 & 1 & 0 & 3 & 5 & 0 & 0 & 1 & 8 & 0 & \\
\hline$\sim$ & 1 & 158 & 0 & 0 & 0 & 1 & 11 & 0 & 0 & 1 & 8 & 4 & \\
\hline$m$ & 0 & 0 & 256 & 0 & 1 & 2 & 3 & 0 & 0 & 6 & 11 & 1 & \\
\hline$\theta$ & 0 & 0 & 3 & 174 & 0 & 3 & 1 & 0 & 0 & 1 & 13 & 0 & \multirow[t]{2}{*}{-8000} \\
\hline in & 0 & 0 & 2 & 0 & 239 & 0 & 5 & 0 & 0 & 0 & 14 & 0 & \\
\hline 0 & 0 & 0 & 0 & 0 & 0 & 7236 & 6 & 0 & 1 & 0 & 0 & 0 & \multirow[t]{2}{*}{-6000} \\
\hline$r$ & 6 & 5 & 4 & 6 & 2 & 8 & 8821 & 2 & 2 & 1 & 0 & 0 & \\
\hline$\infty$ & 0 & 0 & 0 & 0 & 0 & 0 & 0 & 7232 & 0 & 0 & 0 & 0 & \multirow{2}{*}{-4000} \\
\hline a & 0 & 0 & 0 & 0 & 0 & 3 & 15 & 2 & 218 & 0 & 0 & 0 & \\
\hline 욕 & 0 & 0 & 0 & 0 & 0 & 13 & 0 & 0 & 0 & 3538 & 0 & 0 & \multirow{2}{*}{-2000} \\
\hline F & 4 & 2 & 15 & 5 & 2 & 1 & 0 & 0 & 1 & 1 & 11514 & 0 & \\
\hline సి & 0 & 6 & 0 & 0 & 0 & 0 & 0 & 0 & 0 & 2 & 0 & 384 & \\
\hline
\end{tabular}

Figura 18 - Matriz de confusão - Gradient Boost Machine com OvR

As quantidades distribuídas nas matrizes das Figuras 15 e 16 são menores do que as distribuídas nas Figuras 17 e 18, devido ao processo de sampling por segundo sofrido pelos dados, que possibilitou a aplicabilidade nos modelos neurais recorrentes.

A partir das matrizes apresentadas, observa-se o quão impactante é desbalanceamento na quantidade de exemplos para cada classe na classificação das atividades pelos modelos. Inevitavelmente, isto agrega maior desafio para os modelos extraírem padrões para classes com menor quantidade de exemplos associados.

\subsubsection{Desempenho}

A Tabela 4 descreve a taxa de precisão dos modelos para cada atividade desempenhada. O modelo LSTM Bidirecional variou entre 30,77\% (atividade 5) e 100\% (atividade 12) de precisão, com média de 76,91\%. Já o modelo GRU Bidirecional obteve a menor taxa de 29,41\% (atividade 2), a máxima de 98,07\% (atividade 8 ) e média 
de precisão de $73,31 \%$. O modelo Random Forest com OvR obteve precisões entre $91,76 \%$ (atividade 3) e 99,96\% (atividade 8) com média em $97,45 \%$, enquanto que o Gradient Boost Machine com OvR variou com mínimo de 91,43\% (atividade 3), máximo de $99,95 \%$ e média de $97,07 \%$.

Tabela 4 - Taxa de precisão dos modelos por atividade

\begin{tabular}{ccccc}
\hline Atividade & Bi-LSTM & Bi-GRU & RF+OvR & GBM+OvR \\
\hline 1 & 0.875000 & 0.428571 & 0.956044 & 0.945000 \\
2 & 0.800000 & 0.294118 & 0.956522 & 0.923977 \\
3 & 0.333333 & 0.500000 & 0.917603 & 0.914286 \\
4 & 0.555556 & 0.750000 & 0.945055 & 0.935484 \\
5 & 0.307692 & 0.333333 & 0.971074 & 0.979508 \\
6 & 0.973995 & 0.967059 & 0.991780 & 0.995323 \\
7 & 0.904398 & 0.888469 & 0.994921 & 0.994812 \\
8 & 0.976526 & 0.980676 & 0.999585 & 0.999447 \\
9 & 0.583333 & 0.800000 & 0.981735 & 0.981982 \\
10 & 0.951691 & 0.925234 & 0.992701 & 0.996339 \\
11 & 0.967442 & 0.975000 & 0.994561 & 0.995332 \\
12 & 1.000000 & 0.954545 & 0.992327 & 0.987147 \\
\hline
\end{tabular}

A Tabela 5 apresenta a taxa de sensibilidade dos modelos para cada atividade. Aqui, o LSTM Bidirecional varia de $16,67 \%$ (atividade 3) a 99,04\% (atividade 6) de sensibilidade e média de 65,17\%, enquanto o GRU Bidirecional desempenha 16,67\% (atividade 3) e 98,80\% (atividade 6) com média de 62,95\%. O Random Forest com OvR obtém taxas de sensibilidade entre $83,70 \%$ (atividade 2) e 100\% (atividade 8) com média em 93,50\%, e o Gradient Boost Machine com OvR apresenta a menor taxa de $85,87 \%$ (atividade 2), máxima de $100 \%$ (atividade 8 ) e média de $94,85 \%$. Isto reforça o fato de que os modelos de deep learning só acertam muito bem para classes com muitos exemplos de dados, diferente dos algoritmos baseados em árvore onde a discrepância das taxas entre as classes é bem menor.

A Tabela 8 apresenta os valores da métrica de especificidade para cada modelo proposto neste trabalho. Observa-se as variações de taxa entre $97,14 \%$ (atividade 7) e $100 \%$ (atividade 12) para o LSTM Bidirecional; 96,61\% (atividade 7) e 99,95\% (atividades 9 e 12) para o GRU Bidirecional; $99,78 \%$ (atividade 11) e 99,99\% (atividades 8, 9 e 12) para o Random Forest com OvR; e 99,81\% (atividade 11) e 99,99\% (atividade 9) para o Gradient Boost Machine com OvR. As médias para cada modelo são 99,42\%, 99,34\%, 99,93\% e 99,94\%, respectivamente.

Também foram realizados testes de hipótese, para verificar se as taxas de especificidade são estatisticamente melhores entre os modelos baseados em árvore de decisão. Após treinar 30 vezes e coletar os resultados dos modelos, foi aplicado o teste Wilcoxon, no qual verificou-se que o Random Forest com OvR é estatisticamente meIhor na métrica especificidade para as atividades 1,2, 3, 4 e 8 , enquanto que para 
Tabela 5 - Taxa de sensibilidade dos modelos por atividade

\begin{tabular}{ccccc}
\hline Atividade & Bi-LSTM & Bi-GRU & RF+OvR & GBM+OvR \\
\hline 1 & 0.500000 & 0.214286 & 0.840580 & 0.913043 \\
2 & 0.333333 & 0.416667 & 0.836957 & 0.858696 \\
3 & 0.166667 & 0.166667 & 0.875000 & 0.914286 \\
4 & 0.333333 & 0.400000 & 0.882051 & 0.892308 \\
5 & 0.400000 & 0.400000 & 0.903846 & 0.919231 \\
6 & 0.990385 & 0.987981 & 0.999448 & 0.999034 \\
7 & 0.967280 & 0.961145 & 0.995258 & 0.995935 \\
8 & 0.960739 & 0.937644 & 1.000000 & 1.000000 \\
9 & 0.350000 & 0.200000 & 0.903361 & 0.915966 \\
10 & 0.942584 & 0.947368 & 0.995776 & 0.996339 \\
11 & 0.967442 & 0.967442 & 0.997921 & 0.997315 \\
12 & 0.909091 & 0.954545 & 0.989796 & 0.979592 \\
\hline
\end{tabular}

o Gradient Boost Machine com OvR foi confirmado de que é estatisticamente melhor na métrica de especificidade para as atividades 5, 6, 9, 10 e 11. As Tabelas 6 e 7 mostram as atividades e os $\mathrm{p}$-valores resultantes do teste para ambos os modelos.

Tabela 6 - Resultados do Teste Wilcoxon para o modelo Random Forest com OvR

\begin{tabular}{cl}
\hline Atividade & p-valor \\
\hline 1 & $4.230719161336785 \mathrm{e}-06$ \\
2 & $4.4574477483167904 \mathrm{e}-05$ \\
3 & $8.671988141602892 \mathrm{e}-07$ \\
4 & 0.00038548449323139595 \\
7 & 0.8895014228529854 \\
8 & $9.562729679697942 \mathrm{e}-07$ \\
9 & 0.9979444646253911 \\
\hline
\end{tabular}

Tabela 7 - Resultados do Teste Wilcoxon para o modelo Gradient Boost Machine com OvR

\begin{tabular}{cl}
\hline Atividade & p-valor \\
\hline 5 & $8.644742180097877 \mathrm{e}-07$ \\
6 & $8.671988141602892 \mathrm{e}-07$ \\
9 & 0.002055535374608846 \\
10 & $8.671988141602892 \mathrm{e}-07$ \\
11 & $8.671988141602892 \mathrm{e}-07$ \\
\hline
\end{tabular}

A Tabela 9 apresenta o F1-score dos modelos por atividade. As variações são de $22,22 \%$ (atividade 3) a $98,21 \%$ (atividade 6) para o LSTM Bidirecional; $25 \%$ (atividade 3) a $97,74 \%$ (atividade 6) para o GRU Bidirecional; 89,28\% (atividade 2) a 99,98\% (atividade 8) para o Random Forest com OvR; e 89,01\% (atividade 2) a 99,97\% (atividade 8) para o Gradient Boost Machine com OvR. 
Tabela 8 - Taxa de especificidade dos modelos por atividade

\begin{tabular}{ccccc}
\hline Atividade & Bi-LSTM & Bi-GRU & RF+OvR & GBM+OvR \\
\hline 1 & 0.999538 & 0.998144 & 0.999799 & 0.999723 \\
2 & 0.999539 & 0.994447 & 0.999824 & 0.999673 \\
3 & 0.998159 & 0.999071 & 0.999446 & 0.999396 \\
4 & 0.998157 & 0.999070 & 0.999749 & 0.999698 \\
5 & 0.995864 & 0.996293 & 0.999824 & 0.999874 \\
6 & 0.993785 & 0.992032 & 0.998168 & 0.998962 \\
7 & 0.971396 & 0.966150 & 0.998556 & 0.998525 \\
8 & 0.994334 & 0.995444 & 0.999908 & 0.999878 \\
9 & 0.997695 & 0.999535 & 0.999899 & 0.999899 \\
10 & 0.994960 & 0.991886 & 0.999286 & 0.999643 \\
11 & 0.986607 & 0.989651 & 0.997787 & 0.998105 \\
12 & 1.000000 & 0.999531 & 0.999924 & 0.999874 \\
\hline
\end{tabular}

O F1-score geral calculado com base nas de médias de precisão e sensibilidade de cada modelo foi de 70,56\% para o LSTM Bidirecional, 67,73\% para o GRU Bidirecional, 95,43\% para o Random Forest com OvR e 95,94\% para o Gradient Boost Machine com OvR.

Tabela 9 - F1-score dos modelos por atividade

\begin{tabular}{ccccc}
\hline Atividade & Bi-LSTM & Bi-GRU & RF+OvR & GBM+OvR \\
\hline 1 & 0.636364 & 0.285714 & 0.894602 & 0.928747 \\
2 & 0.470588 & 0.344828 & 0.892754 & 0.890141 \\
3 & 0.222222 & 0.250000 & 0.895795 & 0.914286 \\
4 & 0.416667 & 0.521739 & 0.912467 & 0.913386 \\
5 & 0.347826 & 0.363636 & 0.936255 & 0.948413 \\
6 & 0.982122 & 0.977408 & 0.995599 & 0.997175 \\
7 & 0.934783 & 0.923379 & 0.995089 & 0.995374 \\
8 & 0.968568 & 0.958678 & 0.999793 & 0.999724 \\
9 & 0.437500 & 0.320000 & 0.940919 & 0.947826 \\
10 & 0.947115 & 0.936170 & 0.994236 & 0.996339 \\
11 & 0.967442 & 0.971206 & 0.996238 & 0.996322 \\
12 & 0.952381 & 0.954545 & 0.991060 & 0.983355 \\
\hline
\end{tabular}

Por fim, a Tabela 10 traz uma visão geral dos resultados.

Tabela 10 - Taxas gerais das métricas por modelo

\begin{tabular}{cccccc}
\hline Modelo & Acurácia & Precisão $_{\mu}$ & Recall $_{\mu}$ & Especificidade $_{\mu}$ & F1-score \\
\hline Bi-LSTM & 0.940357 & 0.769080 & 0.651738 & 0.994170 & 0.705564 \\
Bi-GRU & 0.931650 & 0.733084 & 0.629479 & 0.993438 & 0.677342 \\
RF+OvR & 0.993579 & 0.974492 & 0.934999 & 0.999347 & 0.954338 \\
GBM+OvR & 0.994401 & 0.970720 & 0.948479 & 0.999437 & 0.959470 \\
\hline
\end{tabular}




\subsection{Discussão}

O Random Forest com OvR se destaca por ser o modelo mais preciso entre os 4 para a maioria das classes (Tabela 4). As diferenças de porcentagem em comparação ao Gradient Boost Machine com OvR são menores que 1,2\%, com exceção da atividade 2 em que alcança a casa dos 3\%. Já em comparação aos modelos de redes neurais, essa diferença chega a $66 \%$. Para a atividade 12 , o modelo LSTM Bidirecional conseguiu obter $100 \%$ de precisão.

Em relação a sensibilidade (Tabela 5), o Gradient Boost Machine com OvR supera com diferenças de até $7 \%$ comparado ao segundo colocado, o Random Forest com OvR. O Random Forest com OvR chega a empatar com o Gradient Boost Machine com OvR em 100\% de sensibilidade na atividade 8, porém entre as atividades 6 e 11, a diferença é insignificante (menor que 0,1\%). Para a atividade 12, a diferença entre ambos é de $1 \%$.

Em relação a métrica de especificidade (Tabela 8), ambos os modelos Random Forest com OvR e Gradient Boost Machine com OvR mantiveram taxas acima de $99,9 \%$. O destaque negativo fica para os modelos de redes neurais, que alcançaram taxas de até $2,7 \%$ a menos do que os outros modelos na atividade 7 . Na atividade 11 , esta diferença é de $1 \%$.

Por fim, os melhores F1-scores ficaram por conta do modelo Gradient Boost Machine com OvR em 9 das 12 atividades classificadas (Tabela 9).

Comparado ao Random Forest com OvR, o modelo obteve as maiores taxas de melhoria para as atividades 3 e 5, superando em 1,8\% e 1,2\% respectivamente. Já para as atividades 2, 8 e 12 o modelo foi pior, porém regredindo menos que $0,8 \%$.

Em relação aos modelos neurais, tanto o Random Forest com OvR quanto o Gradient Boost Machine com OvR foram muito superiores, para todas as atividades. Comparado ao pior resultado (Atividade 3 do LSTM Bidirecional), o Random Forest com OvR supera em taxa 67\% e o Gradient Boost Machine com OvR em 69\%. Comparado ao melhor resultado (Atividade 6 do LSTM Bidirecional), o Random Forest com OvR supera em taxa 1,3\% e o Gradient Boost Machine com OvR em 1,5\%. 


\section{Conclusão e trabalhos futuros}

A diminuição de lesões e mortes causadas por quedas tornaram-se foco de políticas de prevenção, incluindo entre as ações o uso de sistemas de detecção. Através dos dados gerados por sensores de múltiplas modalidades é possível detectar determinados eventos e atividades humanas, onde modelos de aprendizagem de máquina tornaram-se uma alternativa para classificá-los.

Este trabalho de pesquisa buscou elencar modelos de aprendizado de máquina capazes de classificar bem atividades humanas, com base em dados de sensores de múltiplas modalidades. Estes dados foram coletados e disponibilizados publicamente pela Universidad Panamericana para o desafio Challenge UP, que apesar de focar bastante em tipos de quedas também abrange outros tipos de atividades mais comuns como sentar, andar, pular, deitar, e etc.

Foram propostos quatro modelos, sendo dois baseados em redes neurais artificiais (Bi-LSTM e Bi-GRU) e os outros dois baseados em árvores de decisão (Random Forest com OvR, e Gradient Boost Machine com OvR). No geral todos alcançaram bons resultados para a maioria dos dados assistidos, alcançando acurácia acima dos $93 \%$ e F1-score de até quase $96 \%$. Tratando-se dos resultados por atividade, os números são ainda mais surpreendentes: F1-score de até quase $100 \%$.

Os modelos neurais Bi-LSTM e Bi-GRU não conseguiram obter resultados satisfatórios em relação as atividades de 1 a 5 , e 9. Porém para as outras classes em que a base dispusera de quantidades maiores de exemplos, os modelos neurais conseguiram um bom desempenho dado o impacto observado nas taxas de acurácia e nas médias de especificidade.

Durante o processo de experimentação, os modelos baseados em árvore de decisão surpreenderam as expectativas, pois não era esperado que algoritmos tradicionais pudessem desempenhar tão quanto ou melhor do que algoritmos de deep learning. Ao final, a mescla entre o conceito de árvores e o intensificador de gradiente levou o Gradient Boost Machine com OvR como o melhor estimador nos comparativos.

Para trabalhos futuros planeja-se explorar novos algoritmos de aprendizado de máquina (supervisionados e não-supervisionados), e o uso de diferentes estimadores para construção de modelos únicos capazes de classificar atividades, mesmo com poucos canais e poucos exemplos disponíveis para aplicação em treinamento. 


\section{Referências}

BALTRUŠAITIS, T.; AHUJA, C.; MORENCY, L.-P. Multimodal machine learning: A survey and taxonomy. IEEE Transactions on Pattern Analysis and Machine Intelligence, IEEE, v. 41, n. 2, p. 423-443, 2019. Citado na página 14.

BISHOP, C. Pattern Recognition and Machine Learning. Springer, 2006. (Information Science and Statistics). ISBN 9780387310732. Disponível em: (https://books.google. com.br/books?id=kTNoQgAACAAJ $\rangle$. Citado na página 23.

BROWNSELL, S.; HAWLEY, M. S. Automatic fall detectors and the fear of falling. Journal of telemedicine and telecare, SAGE Publications Sage UK: London, England, v. 10, n. 5, p. 262-266, 2004. Citado na página 14.

CHALLENGE UP website. 2019. Available at: /https://sites.google.com/up.edu.mx/ challenge-up-2019/overview?authuser=0.Lastaccess:April,2019.) Citado 3 vezes nas páginas 9, 26 e 27.

CHEN, C.; JAFARI, R.; KEHTARNAVAZ, N. A survey of depth and inertial sensor fusion for human action recognition. Multimedia Tools and Applications, Springer, v. 76, n. 3, p. 4405-4425, 2017. Citado na página 14.

CHOLLET, F. Deep Learning with Python. Manning Publications Company, 2017. ISBN 9781617294433. Disponível em: 〈https://books.google.com.br/books?id= Yo3CAQAACAAJ). Citado 6 vezes nas páginas 17, 18, 19, 20, 21 e 23.

CHUNG, S. et al. Sensor data acquisition and multimodal sensor fusion for human activity recognition using deep learning. Sensors, Multidisciplinary Digital Publishing Institute, v. 19, n. 7, p. 1716, 2019. Citado 2 vezes nas páginas 15 e 24.

DING, L. et al. A deep hybrid learning model to detect unsafe behavior: integrating convolution neural networks and long short-term memory. Automation in Construction, Elsevier, v. 86, p. 118-124, 2018. Citado na página 24.

GOODFELLOW, I. et al. Deep learning. [S.I.]: MIT press Cambridge, 2016. v. 1. Citado 5 vezes nas páginas $9,17,18,21$ e 22.

IGUAL, R.; MEDRANO, C.; PLAZA, I. Challenges, issues and trends in fall detection systems. Biomedical engineering online, BioMed Central, v. 12, n. 1, p. 66, 2013. Citado na página 14.

JEWITT, C. Different approaches to multimodality. In: . [S.I.]: Routledge, 2009. Citado na página 26.

KANJO, E.; YOUNIS, E. M.; ANG, C. S. Deep learning analysis of mobile physiological, environmental and location sensor data for emotion detection. Information Fusion, Elsevier, v. 49, p. 46-56, 2019. Citado na página 14.

KINGMA, D. P.; BA, J. Adam: A method for stochastic optimization. arXiv preprint arXiv:1412.6980, 2014. Citado na página 28. 
$\mathrm{LI}, \mathrm{X}$. et al. Fall detection for elderly person care using convolutional neural networks. In: IEEE. 2017 10th International Congress on Image and Signal Processing, BioMedical Engineering and Informatics (CISP-BMEI). [S.I.], 2017. p. 1-6. Citado 2 vezes nas páginas 14 e 23.

Michael Nguyen. Illustrated Guide to LSTM's and GRU's: $A$ step by step explanation. 2018. /https://towardsdatascience.com/ illustrated-guide-to-Istms-and-gru-s-a-step-by-step-explanation-44e9eb85bf21〉, Last accessed on 2019-06-15. Citado 2 vezes nas páginas 9 e 20.

MIOTTO, R. et al. Deep learning for healthcare: review, opportunities and challenges. Briefings in bioinformatics, Oxford University Press, v. 19, n. 6, p. 1236-1246, 2017. Citado na página 14.

NEVEROVA, N. et al. Learning human identity from motion patterns. IEEE Access, IEEE, v. 4, p. 1810-1820, 2016. Citado na página 24.

NGIAM, J. et al. Multimodal deep learning. In: Proceedings of the 28th international conference on machine learning (ICML-11). [S.I.: s.n.], 2011. p. 689-696. Citado na página 14.

NÚÑEZ, J. C. et al. Convolutional neural networks and long short-term memory for skeleton-based human activity and hand gesture recognition. Pattern Recognition, Elsevier, v. 76, p. 80-94, 2018. Citado na página 25.

NWEKE, H. F. et al. Data fusion and multiple classifier systems for human activity detection and health monitoring: Review and open research directions. Information Fusion, Elsevier, v. 46, p. 147-170, 2019. Citado na página 14.

ORDÓÑEZ, F.; ROGGEN, D. Deep convolutional and Istm recurrent neural networks for multimodal wearable activity recognition. Sensors, Multidisciplinary Digital Publishing Institute, v. 16, n. 1, p. 115, 2016. Citado 2 vezes nas páginas 14 e 25.

ORGANIZATION, W. H.; AGEING, W. H. O.; UNIT, L. C. WHO global report on falls prevention in older age. [S.I.]: World Health Organization, 2007. Citado na página 14.

ROUAST, P. V.; ADAM, M.; CHIONG, R. Deep learning for human affect recognition: Insights and new developments. IEEE Transactions on Affective Computing, IEEE, 2019. Citado na página 14.

SCHEFFER, A. C. et al. Fear of falling: measurement strategy, prevalence, risk factors and consequences among older persons. Age and ageing, Oxford University Press, v. 37, n. 1, p. 19-24, 2008. Citado na página 14.

SHALEV-SHWARTZ, S.; BEN-DAVID, S. Understanding machine learning: From theory to algorithms. [S.I.]: Cambridge university press, 2014. Citado 2 vezes nas páginas 21 e 23.

SUN, S.-W. et al. Baseball player behavior classification system using long short-term memory with multimodal features. Sensors, Multidisciplinary Digital Publishing Institute, v. 19, n. 6, p. 1425, 2019. Citado 2 vezes nas páginas 15 e 24.

WANG, J. et al. Deep learning for sensor-based activity recognition: A survey. Pattern Recognition Letters, Elsevier, v. 119, p. 3-11, 2019. Citado na página 23. 
WORLD Health Organization website. 2018. Available at: /https://www.who.int/ news-room/fact-sheets/detail/falls.Lastaccess:June,2019.) Citado na página 14.

YANG, J. et al. Deep convolutional neural networks on multichannel time series for human activity recognition. In: Twenty-Fourth International Joint Conference on Artificial Intelligence. [S.I.: s.n.], 2015. Citado na página 24.

ZHAO, K.; CHU, W.-S.; ZHANG, H. Deep region and multi-label learning for facial action unit detection. In: Proceedings of the IEEE Conference on Computer Vision and Pattern Recognition. [S.I.: s.n.], 2016. p. 3391-3399. Citado na página 28. 
Anexos 


\section{ANEXO A - UP-Fall Detection Dataset: Dispositivos e Canais}

\begin{tabular}{|c|c|c|}
\hline Dispositivo & Canal & Unidade \\
\hline \multirow{7}{*}{ Vestível do tornozelo } & Acelerômetro (eixo x) & $g$ \\
\hline & Acelerômetro (eixo y) & g \\
\hline & Acelerômetro (eixo z) & g \\
\hline & Giroscópio (rolamento) & $\mathrm{deg} / \mathrm{s}$ \\
\hline & Giroscópio (inclinação) & $\mathrm{deg} / \mathrm{s}$ \\
\hline & Giroscópio (guinada) & $\mathrm{deg} / \mathrm{s}$ \\
\hline & Luminosidade & $\operatorname{lux}$ \\
\hline \multirow{7}{*}{ Vestível do bolso direito } & Acelerômetro (eixo x) & g \\
\hline & Acelerômetro (eixo y) & $g$ \\
\hline & Acelerômetro (eixo z) & $\mathrm{g}$ \\
\hline & Giroscópio (rolamento) & $\mathrm{deg} / \mathrm{s}$ \\
\hline & Giroscópio (inclinação) & $\mathrm{deg} / \mathrm{s}$ \\
\hline & Giroscópio (guinada) & $\mathrm{deg} / \mathrm{s}$ \\
\hline & Luminosidade & $\operatorname{lux}$ \\
\hline \multirow{7}{*}{ Vestível da cintura } & Acelerômetro (eixo x) & $\mathrm{g}$ \\
\hline & Acelerômetro (eixo y) & g \\
\hline & Acelerômetro (eixo z) & $\mathrm{g}$ \\
\hline & Giroscópio (rolamento) & $\mathrm{deg} / \mathrm{s}$ \\
\hline & Giroscópio (inclinação) & $\mathrm{deg} / \mathrm{s}$ \\
\hline & Giroscópio (guinada) & $\mathrm{deg} / \mathrm{s}$ \\
\hline & Luminosidade & $\operatorname{lux}$ \\
\hline \multirow{7}{*}{ Vestível do pescoço } & Acelerômetro (eixo x) & $g$ \\
\hline & Acelerômetro (eixo y) & $\mathrm{g}$ \\
\hline & Acelerômetro (eixo z) & $\mathrm{g}$ \\
\hline & Giroscópio (rolamento) & $\mathrm{deg} / \mathrm{s}$ \\
\hline & Giroscópio (inclinação) & $\mathrm{deg} / \mathrm{s}$ \\
\hline & Giroscópio (guinada) & $\mathrm{deg} / \mathrm{s}$ \\
\hline & Luminosidade & $\operatorname{lux}$ \\
\hline \multirow{7}{*}{ Vestível do pulso } & Acelerômetro (eixo x) & $\mathrm{g}$ \\
\hline & Acelerômetro (eixo y) & g \\
\hline & Acelerômetro (eixo z) & $\mathrm{g}$ \\
\hline & Giroscópio (rolamento) & $\mathrm{deg} / \mathrm{s}$ \\
\hline & Giroscópio (inclinação) & $\mathrm{deg} / \mathrm{s}$ \\
\hline & Giroscópio (guinada) & $\mathrm{deg} / \mathrm{s}$ \\
\hline & Luminosidade & $\operatorname{lux}$ \\
\hline Headset EEG & Sinal de onda cerebral & $\mu \mathrm{V}$ \\
\hline Infravermelho 1 & Sem interrupção & falso(0)/verdadeiro(1) \\
\hline Infravermelho 2 & Sem interrupção & falso(0)/verdadeiro(1) \\
\hline Infravermelho 3 & Sem interrupção & falso(0)/verdadeiro(1) \\
\hline Infravermelho 4 & Sem interrupção & falso(0)/verdadeiro(1) \\
\hline Infravermelho 5 & Sem interrupção & falso(0)/verdadeiro(1) \\
\hline Infravermelho 6 & Sem interrupção & falso(0)/verdadeiro(1) \\
\hline Câmera 1 & Visualização lateral & $640 x 480 \mathrm{px}$ \\
\hline Câmera 2 & Visualização frontal & $640 x 480 \mathrm{px}$ \\
\hline
\end{tabular}

